\title{
Preparation of Bifunctional Allylboron Reagent and Application to Aldehyde Allylboration
}

Jiaming Liu, Shang Gao and Ming Chen*

Department of Chemistry and Biochemistry

Auburn University

E-mail: mzc0102@auburn.edu

Supporting Information: Experimental Procedures, Tabulated Spectroscopic Data, ${ }^{1} \mathrm{H}$ and

${ }^{13} \mathrm{C}$ Spectra of New Compounds

SI-1 
General Experimental Details. All reaction solvents were purified before use. Tetrahydrofuran, diethyl ether and toluene were purified by passing through a solvent column composed of activated A-1 alumina. Unless indicated otherwise, all reactions were conducted under an atmosphere of argon using flame-dried or oven-dried $\left(120{ }^{\circ} \mathrm{C}\right)$ glassware. The term "concentrated under reduced pressure" refers to the removal of solvents and other volatile materials using a rotary evaporator with the water bath temperature below $30{ }^{\circ} \mathrm{C}$, followed by the removal of residual solvents at high vacuum $(<$ 0.2 mbar).

Proton nuclear magnetic resonance ( ${ }^{1} \mathrm{H}$ NMR) spectra were acquired on commercial instruments (400 and $600 \mathrm{MHz}$ ) at Auburn University NMR facility. Carbon-13 nuclear magnetic resonance $\left({ }^{13} \mathrm{C}\right.$ NMR $)$ spectra were acquired at 101 and $151 \mathrm{MHz}$. The proton signal for the residual non-deuterated solvent ( $\delta 7.26$ for chloroform, $\delta 2.05$ for acetone and $\delta 2.08$ for toluene) was used as an internal reference for ${ }^{1} \mathrm{H}$ NMR spectra. For ${ }^{13} \mathrm{C}$ NMR spectra, chemical shifts are reported relative to the resonance of chloroform $(\delta$ 77.36) and acetone $(\delta 29.84)$. Coupling constants are reported in Hz. High-resolution mass spectra were recorded on a commercial high-resolution mass spectrometer via the Micro Mass/Analytical Facility operated by the College of Chemistry and Biochemistry, Auburn University.

Analytical thin layer chromatography (TLC) was performed on Kieselgel 60 F254 glass plates precoated with a $0.25 \mathrm{~mm}$ thickness of silica gel. The TLC plates were visualized with UV light and/or by staining with Hanessian solution (ceric sulfate and ammonium molybdate in aqueous sulfuric acid) or $\mathrm{KMnO}_{4}$. Column chromatography was generally performed using Kieselgel 60 (230-400 mesh) silica gel, typically using a 50-100:1 weight ratio of silica gel to crude product. 


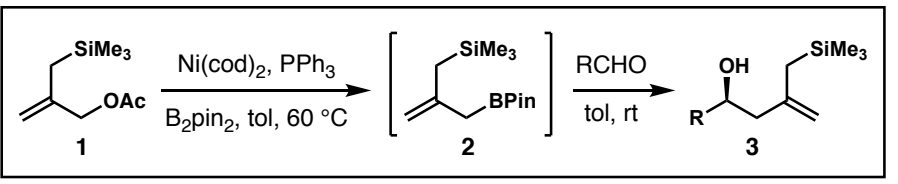

General procedure for synthesis of homoallylic alcohols 3: In an Ar-filled glove box, allylic acetate 1 (56 mg, $0.3 \mathrm{mmol}, 1$ equiv), $\mathrm{B}_{2} \mathrm{Pin}_{2}$ (84 mg, $0.33 \mathrm{mmol}, 1.1$ equiv), $\mathrm{Ni}(\mathrm{cod})_{2}$ (4 mg, $\left.0.015 \mathrm{mmol}, 5 \mathrm{~mol} \%\right), \mathrm{PPh}_{3}(4 \mathrm{mg}, 0.015 \mathrm{mmol}, 5 \mathrm{~mol} \%)$, toluene $(0.5$ $\mathrm{mL}$ ) and a Teflon-coated magnetic stirring bar were sequentially added into a 1-dram vial. The vial was sealed with a cap containing a PTFE-lined silicone septum and removed from the glove box stirring at $60{ }^{\circ} \mathrm{C}$ for $2 \mathrm{~h}$. After complete consumption of allylic acetate 1, the mixture was filtered through a short pad of Celite and the resulting solution was concentrated under reduced pressure. Then the obtained allylboronate 2 was dissolved in anhydrous toluene and was used for aldehyde allylation without further purification. To a reaction vial containing a Teflon-coated magnetic stirring bar was added freshly distilled aldehyde ( $0.1 \mathrm{mmol}, 1.0$ equiv) followed by addition of allylboronate $2(0.15 \mathrm{mmol}, 0.25$ $\mathrm{mL}$ of a $0.6 \mathrm{M}$ solution in toluene). The reaction mixture was allowed to stir at ambient temperature. After complete consumption of the aldehyde, purification of the crude reaction mixture was performed by flash chromatography (gradient elution with hexane and EtOAc) to provide alcohol 3.

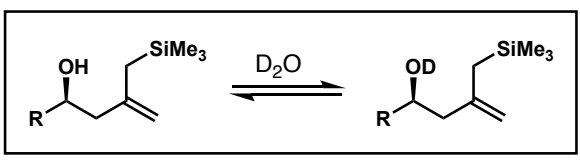

When $\left(\mathrm{CD}_{3}\right)_{2} \mathrm{CO}$ was used as the solvent for NMR analysis, partial hydrogen-deuterium exchange between the alcohol $\mathrm{OH}$ group and the residue $\mathrm{D}_{2} \mathrm{O}$ in $\left(\mathrm{CD}_{3}\right)_{2} \mathrm{CO}$ was observed in both ${ }^{1} \mathrm{H}$ NMR and ${ }^{13} \mathrm{C}$ NMR. Particularly in ${ }^{13} \mathrm{C}$ NMR, two signals (for one carbon) were observed for several carbon atoms (for an example, see page 16). The H-D exchange was further confirmed by the results obtained from adding additional $10 \mu \mathrm{L}$ $\mathrm{D}_{2} \mathrm{O}$ to the NMR sample. In this case, full H-D exchange was observed. Only one set of carbon signals were detected in ${ }^{13} \mathrm{C}$ NMR spectra. 


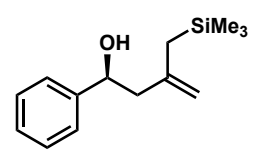

rac-1-phenyl-3-((trimethylsilyl)methyl)but-3-en-1-ol (3a) Prepared according to the general procedure. The crude mixture was purified by column chromatography to give compound $\mathbf{3 a}$ in $73 \%$ yield $(17 \mathrm{mg})$ as colorless oil. ${ }^{1} \mathrm{H}$ NMR (600 MHz, $\left.\mathrm{CDCl}_{3}\right) \delta 7.30-7.43(\mathrm{~m}, 4 \mathrm{H}), 7.19-7.30(\mathrm{~m}, 1 \mathrm{H})$, $4.77(\mathrm{~s}, 2 \mathrm{H}), 4.73(\mathrm{~s}, 1 \mathrm{H}), 2.21-2.43(\mathrm{~m}, 3 \mathrm{H}), 1.61(\mathrm{~d}, J=13.9 \mathrm{~Hz}, 1 \mathrm{H}), 1.56(\mathrm{~d}, J=$ $13.4 \mathrm{~Hz}, 1 \mathrm{H}), 0.02$ (s, 9H). ${ }^{13} \mathrm{C} \mathrm{NMR}\left(151 \mathrm{MHz}, \mathrm{CDCl}_{3}\right) \delta 144.7,144.2,128.7,127.8$, 126.1, 111.5, 71.3, 49.5, 26.7, -1.0. HRMS (ESI): $\mathrm{m} / \mathrm{z}$ for $\mathrm{C}_{14} \mathrm{H}_{22} \mathrm{OSiNa}[\mathrm{M}+\mathrm{Na}]^{+}$calcd. 257.1338, found: 257.1334 .

(3b) Prepared according to the general procedure. The crude mixture
was purified by column chromatography to give compound $\mathbf{3 b}$ in $78 \%$ yield $(21 \mathrm{mg})$ as colorless oil. ${ }^{1} \mathrm{H}$ NMR $\left(400 \mathrm{MHz}, \mathrm{CDCl}_{3}\right) \delta 7.30(\mathrm{~d}, J=8.5 \mathrm{~Hz}, 2 \mathrm{H})$, $6.89(\mathrm{~d}, J=8.6 \mathrm{~Hz}, 2 \mathrm{H}), 4.77(\mathrm{~s}, 1 \mathrm{H}), 4.74-4.76(\mathrm{~m}, 1 \mathrm{H}), 4.73(\mathrm{~s}, 1 \mathrm{H}), 3.81(\mathrm{~s}, 3 \mathrm{H})$, $2.34(\mathrm{~d}, J=6.7 \mathrm{~Hz}, 2 \mathrm{H}), 2.21(\mathrm{~s}, 1 \mathrm{H}), 1.63(\mathrm{~d}, J=13.4 \mathrm{~Hz}, 1 \mathrm{H}), 1.57$ (d, $J=14.4 \mathrm{~Hz}, 1 \mathrm{H})$, 0.04 (s, 9H). ${ }^{13} \mathrm{C}$ NMR (151 MHz, $\left.\mathrm{CDCl}_{3}\right) \delta 159.2,144.8,136.4,127.3,114.0,111.4$, 71.0, 55.6, 49.3, 26.7, -1.0. HRMS (ESI): $\mathrm{m} / \mathrm{z}$ for $\mathrm{C}_{15} \mathrm{H}_{24} \mathrm{O}_{2} \mathrm{SiNa}[\mathrm{M}+\mathrm{Na}]^{+}$calcd. 287.1443, found: 287.1450 .

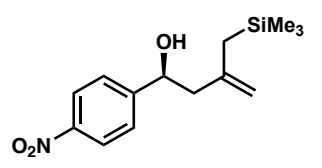

rac-1-(4-nitrophenyl)-3-((trimethylsilyl)methyl)but-3-en-1-ol (3c) Prepared according to the general procedure. The crude mixture was purified by column chromatography to give compound $3 \mathrm{c}$ in $77 \%$ yield $(22 \mathrm{mg})$ as colorless oil. ${ }^{1} \mathrm{H} \mathrm{NMR}\left(600 \mathrm{MHz}, \mathrm{CDCl}_{3}\right) \delta 8.21(\mathrm{~d}, J=8.6 \mathrm{~Hz}, 2 \mathrm{H})$, $7.55(\mathrm{~d}, J=8.5 \mathrm{~Hz}, 2 \mathrm{H}), 4.84-4.94(\mathrm{~m}, 1 \mathrm{H}), 4.80(\mathrm{~s}, 2 \mathrm{H}), 2.49(\mathrm{~s}, 1 \mathrm{H}), 2.37$ (dd, $J=$ 10.2, $6.0 \mathrm{~Hz}, 1 \mathrm{H}), 2.25(\mathrm{dd}, J=13.5,10.2 \mathrm{~Hz}, 1 \mathrm{H}), 1.63$ (d, $J=13.4 \mathrm{~Hz}, 1 \mathrm{H}), 1.56(\mathrm{~d}, J=$ $13.4 \mathrm{~Hz}, 1 \mathrm{H}), 0.05$ (s, 9H). ${ }^{13} \mathrm{C} \mathrm{NMR}\left(151 \mathrm{MHz}, \mathrm{CDCl}_{3}\right) \delta 151.7,147.4,143.8,126.8$, 124.0, 112.4, 70.3, 49.5, 26.6, -1.1. HRMS (ESI): $\mathrm{m} / \mathrm{z}$ for $\mathrm{C}_{14} \mathrm{H}_{21} \mathrm{NO}_{3} \mathrm{SiNa}[\mathrm{M}+\mathrm{Na}]^{+}$ calcd. 302.1188, found: 302.1187.

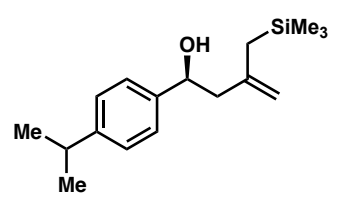

rac-1-(4-isopropylphenyl)-3-((trimethylsilyl)methyl)but-3-en-1-o I (3d) Prepared according to the general procedure. The crude mixture was purified by column chromatography to give compound 3d in $92 \%$ yield $(25 \mathrm{mg})$ as colorless oil. ${ }^{1} \mathrm{H}$ NMR $(600 \mathrm{MHz}$, $d^{6}$-acteone) $\delta 7.29(\mathrm{~d}, J=7.8 \mathrm{~Hz}, 2 \mathrm{H}), 7.19(\mathrm{~d}, J=7.8 \mathrm{~Hz}, 2 \mathrm{H}), 4.74-4.84(\mathrm{~m}, 1 \mathrm{H}), 4.66$ $(\mathrm{s}, 1 \mathrm{H}), 4.58(\mathrm{~s}, 1 \mathrm{H}), 3.93(\mathrm{~s}, 1 \mathrm{H}), 2.80-2.97(\mathrm{~m}, 1 \mathrm{H}), 2.37(\mathrm{dd}, J=13.5,8.9 \mathrm{~Hz}, 1 \mathrm{H})$, $2.33-2.20(\mathrm{~m}, 1 \mathrm{H}), 1.65(\mathrm{~d}, J=13.4 \mathrm{~Hz}, 1 \mathrm{H}), 1.59$ (d, $J=13.4 \mathrm{~Hz}, 1 \mathrm{H}), 1.21$ (d, $J=6.9$ 
$\mathrm{Hz}, 6 \mathrm{H}), 0.03$ (s, 9H). ${ }^{13} \mathrm{C}$ NMR (151 MHz, $d^{6}$-acteone) $\delta 148.0,145.5,144.3,126.74$, 126.70, 110.27 (110.26), 72.6 (72.5), 49.5 (49.4), 34.5, 27.1, 24.4, -1.3. HRMS (ESI): $\mathrm{m} / \mathrm{z}$ for $\mathrm{C}_{17} \mathrm{H}_{28} \mathrm{OSiNa}[\mathrm{M}+\mathrm{Na}]^{+}$calcd. 299.1807, found: 299.1815.

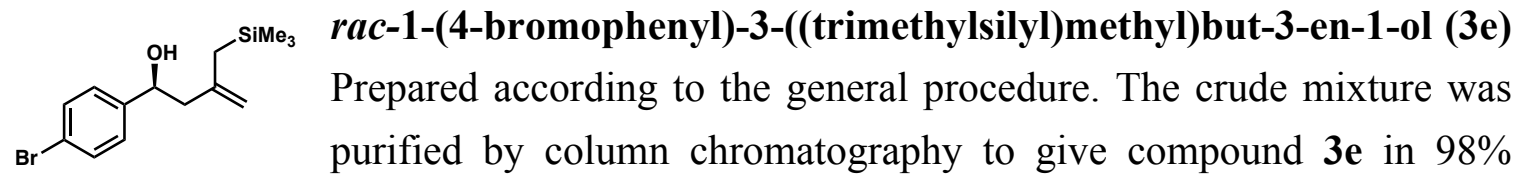
yield $(31 \mathrm{mg})$ as colorless oil. ${ }^{1} \mathrm{H}$ NMR $\left(600 \mathrm{MHz}, d^{6}\right.$-acteone) $\delta 7.49(\mathrm{~d}, J=8.2 \mathrm{~Hz}, 2 \mathrm{H})$, $7.34(\mathrm{~d}, J=8.1 \mathrm{~Hz}, 2 \mathrm{H}), 4.78-4.90(\mathrm{~m}, 1 \mathrm{H}), 4.62(\mathrm{~s}, 1 \mathrm{H}), 4.57(\mathrm{~s}, 1 \mathrm{H}), 4.22(\mathrm{~s}, 1 \mathrm{H})$, $2.38(\mathrm{dd}, J=13.4,8.5 \mathrm{~Hz}, 1 \mathrm{H}), 2.30(\mathrm{dd}, J=13.5,4.0 \mathrm{~Hz}, 1 \mathrm{H}), 1.64(\mathrm{~d}, J=13.4 \mathrm{~Hz}, 1 \mathrm{H})$, $1.59(\mathrm{~d}, J=13.4 \mathrm{~Hz}, 1 \mathrm{H}), 0.03(\mathrm{~s}, 9 \mathrm{H}) .{ }^{13} \mathrm{C} \mathrm{NMR}\left(151 \mathrm{MHz}, d^{6}\right.$-acteone) $\delta 146.2,145.0$, 131.8, 128.9, 120.7, 110.62 (110.60), 72.2 (72.1), 49.41 (49.35), 27.1, -1.3. HRMS (ESI): $\mathrm{m} / \mathrm{z}$ for $\mathrm{C}_{14} \mathrm{H}_{21} \mathrm{BrOSiNa}[\mathrm{M}+\mathrm{Na}]^{+}$calcd. 335.0443, found: 335.0435 .

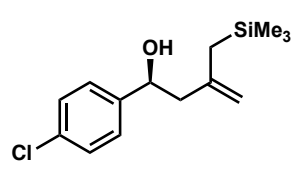

rac-1-(4-chlorophenyl)-3-((trimethylsilyl)methyl)but-3-en-1-ol (3f) Prepared according to the general procedure. The crude mixture was purified by column chromatography to give compound $3 \mathrm{f}$ in $97 \%$ yield $(26 \mathrm{mg})$ as colorless oil. ${ }^{1} \mathrm{H}$ NMR $\left(600 \mathrm{MHz}, d^{6}\right.$-acteone) $\delta 7.40(\mathrm{~d}, J=8.6 \mathrm{~Hz}, 2 \mathrm{H}), 7.34$ $(\mathrm{d}, J=8.4 \mathrm{~Hz}, 2 \mathrm{H}), 4.85$ (dt, $J=8.3,4.4 \mathrm{~Hz}, 1 \mathrm{H}), 4.62(\mathrm{~s}, 1 \mathrm{H}), 4.57(\mathrm{~s}, 1 \mathrm{H}), 4.23(\mathrm{~s}, 1 \mathrm{H})$, $2.39(\mathrm{dd}, J=14.0,8.2 \mathrm{~Hz}, 1 \mathrm{H}), 2.30(\mathrm{dd}, J=14.0,5.2 \mathrm{~Hz}, 1 \mathrm{H}), 1.64$ (d, $J=13.5 \mathrm{~Hz}, 1 \mathrm{H})$, $1.59(\mathrm{~d}, J=13.3 \mathrm{~Hz}, 1 \mathrm{H}), 0.02(\mathrm{~s}, 9 \mathrm{H}) .{ }^{13} \mathrm{C} \mathrm{NMR}\left(151 \mathrm{MHz}, d^{6}\right.$-acteone) $\delta 145.7,145.0$, 132.6, 128.8, 128.5, 110.6, 72.1, 49.4, 27.1, -1.3. HRMS (ESI): $\mathrm{m} / \mathrm{z}$ for $\mathrm{C}_{14} \mathrm{H}_{21} \mathrm{ClOSiNa}$ $[\mathrm{M}+\mathrm{Na}]^{+}$calcd. 291.0948, found: 291.0954.

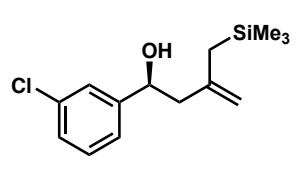

rac-1-(3-chlorophenyl)-3-((trimethylsilyl)methyl)but-3-en-1-ol (3g) Prepared according to the general procedure. The crude mixture was purified by column chromatography to give compound $\mathbf{3 g}$ in $79 \%$ yield $(21 \mathrm{mg})$ as colorless oil. ${ }^{1} \mathrm{H}$ NMR $\left(600 \mathrm{MHz}, d^{6}\right.$-acteone) $\delta 7.42(\mathrm{~s}, 1 \mathrm{H}), 7.29-7.37$ $(\mathrm{m}, 2 \mathrm{H}), 7.25(\mathrm{~d}, J=6.2 \mathrm{~Hz}, 1 \mathrm{H}), 4.81-4.91(\mathrm{~m}, 1 \mathrm{H}), 4.64(\mathrm{~s}, 1 \mathrm{H}), 4.59(\mathrm{~s}, 1 \mathrm{H}), 4.26(\mathrm{~s}$, 1H), 2.39 (dd, $J=13.6,8.4 \mathrm{~Hz}, 1 \mathrm{H}), 2.32(\mathrm{dd}, J=13.7,4.2 \mathrm{~Hz}, 1 \mathrm{H}), 1.65$ (d, $J=13.4 \mathrm{~Hz}$, $1 \mathrm{H}), 1.60(\mathrm{~d}, J=13.4 \mathrm{~Hz}, 1 \mathrm{H}), 0.03(\mathrm{~s}, 9 \mathrm{H}) .{ }^{13} \mathrm{C}$ NMR (151 MHz, $d^{6}$-acteone) $\delta 149.4$, 145.0, 134.2, 130.5, 127.5, 126.7, 125.3, 110.67 (110.65), 72.2 (72.1), 49.4 (49.3), 27.0, -1.3. HRMS (ESI): $\mathrm{m} / \mathrm{z}$ for $\mathrm{C}_{14} \mathrm{H}_{21} \mathrm{ClOSiNa}[\mathrm{M}+\mathrm{Na}]^{+}$calcd. 291.0948, found: 291.0947. 


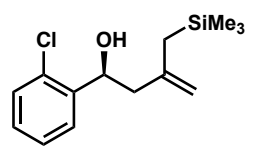

rac-1-(2-chlorophenyl)-3-((trimethylsilyl)methyl)but-3-en-1-ol

Prepared according to the general procedure. The crude mixture was purified by column chromatography to give compound $\mathbf{3 h}$ in $91 \%$ yield $(24 \mathrm{mg})$ as colorless oil. ${ }^{1} \mathrm{H}$ NMR $\left(600 \mathrm{MHz}, d^{8}\right.$-toluene) $\delta 7.69(\mathrm{~d}, J=7.7 \mathrm{~Hz}, 1 \mathrm{H}), 7.06$ $-7.12(\mathrm{~m}, 1 \mathrm{H}), 6.94-7.00(\mathrm{~m}, 1 \mathrm{H}), 6.78(\mathrm{dd}, J=7.7 \mathrm{~Hz}, 7.0 \mathrm{~Hz}, 1 \mathrm{H}), 5.11(\mathrm{~d}, J=9.7$ $\mathrm{Hz}, 1 \mathrm{H}), 4.72$ (s, 1H), 4.63 (s, 1H), 2.51 (d, $J=13.8 \mathrm{~Hz}, 1 \mathrm{H}), 2.00$ (dd, $J=13.7,10.0 \mathrm{~Hz}$, $1 \mathrm{H}), 1.90(\mathrm{~d}, J=2.6 \mathrm{~Hz}, 1 \mathrm{H}), 1.58$ (d, $J=13.4 \mathrm{~Hz}, 1 \mathrm{H}), 1.49$ (d, $J=13.3 \mathrm{~Hz}, 1 \mathrm{H}), 0.00$ (s, 9H). ${ }^{13} \mathrm{C}$ NMR (151 MHz, $d^{6}$-acteone) $\delta 145.1,144.2,131.8,129.7,129.0,128.3,127.9$, 110.4, 69.1, 47.8, 26.9, -1.3. HRMS (ESI): $\mathrm{m} / \mathrm{z}$ for $\mathrm{C}_{14} \mathrm{H}_{21} \mathrm{ClOSiNa}[\mathrm{M}+\mathrm{Na}]^{+}$calcd. 291.0948, found: 291.0945 .

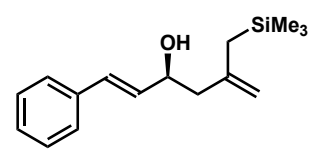

rac-(E)-1-phenyl-5-((trimethylsilyl)methyl)hexa-1,5-dien-3-ol (3i) Prepared according to the general procedure. The crude mixture was purified by column chromatography to give compound $3 \mathbf{i}$ in $67 \%$ yield $(17 \mathrm{mg})$ as colorless oil. ${ }^{1} \mathrm{H}$ NMR $\left(600 \mathrm{MHz}, d^{8}\right.$-toluene) $\delta 7.23(\mathrm{~d}, J=7.6 \mathrm{~Hz}, 2 \mathrm{H})$, $7.06-7.13(\mathrm{~m}, 2 \mathrm{H}), 7.00-7.05(\mathrm{~m}, 1 \mathrm{H}), 6.59(\mathrm{~d}, J=15.9 \mathrm{~Hz}, 1 \mathrm{H}), 6.14(\mathrm{dd}, J=15.9,5.7$ $\mathrm{Hz}, 1 \mathrm{H}), 4.72$ (s, 1H), 4.65 (s, 1H), 4.25 (dt, $J=10.0,6.2 \mathrm{~Hz}, 1 \mathrm{H}), 2.17$ (d, $J=6.6 \mathrm{~Hz}$, 2H), 1.53 (d, $J=3.0 \mathrm{~Hz}, 1 \mathrm{H}), 1.50$ (d, $J=13.4 \mathrm{~Hz}, 1 \mathrm{H}), 1.45$ (d, $J=13.4 \mathrm{~Hz}, 1 \mathrm{H}), 0.00$ (s, 9H). ${ }^{13} \mathrm{C}$ NMR (151 MHz, $d^{6}$-acteone) $\delta$ 145.2, 138.2, 134.3, 129.3(2C), 128.0, 127.1, 110.3, 71.1, 47.4, 27.3, -1.3. HRMS (ESI): $\mathrm{m} / \mathrm{z}$ for $\mathrm{C}_{14} \mathrm{H}_{24} \mathrm{OSiNa}[\mathrm{M}+\mathrm{Na}]^{+}$calcd. 283.1494, found: 283.1512 .

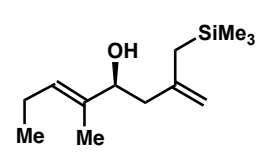

\section{rac-(E)-5-methyl-2-((trimethylsilyl)methyl)octa-1,5-dien-4-ol}

Prepared according to the general procedure. The crude mixture was purified by column chromatography to give compound $\mathbf{3 j}$ in $80 \%$ yield $(18 \mathrm{mg})$ as colorless oil. ${ }^{1} \mathrm{H}$ NMR $\left(400 \mathrm{MHz}, d^{6}\right.$-acteone) $\delta 5.36(\mathrm{t}, J=7.0 \mathrm{~Hz}, 1 \mathrm{H}), 4.65$ (s, 1H), $4.56(\mathrm{~s}, 1 \mathrm{H}), 4.01-4.19(\mathrm{~m}, 1 \mathrm{H}), 3.35(\mathrm{~d}, J=3.6 \mathrm{~Hz}, 1 \mathrm{H}), 2.18(\mathrm{~d}, J=6.6 \mathrm{~Hz}$, 2H), $1.91-2.03(\mathrm{~m}, 2 \mathrm{H}), 1.51-1.73(\mathrm{~m}, 5 \mathrm{H}), 0.92(\mathrm{t}, J=7.5 \mathrm{~Hz}, 3 \mathrm{H}), 0.03(\mathrm{~s}, 9 \mathrm{H}) .{ }^{13} \mathrm{C}$ NMR (151 MHz, $d^{6}$-acteone) $\delta 145.8,138.0,127.4,109.82$ (109.81), 76.2 (76.1), 45.4 (45.3), 27.0, 21.3, 14.4, 11.3, -1.2. HRMS (ESI): $\mathrm{m} / \mathrm{z}$ for $\mathrm{C}_{13} \mathrm{H}_{26} \mathrm{OSiNa}[\mathrm{M}+\mathrm{Na}]^{+}$calcd. 249.1651, found: 249.1647 .

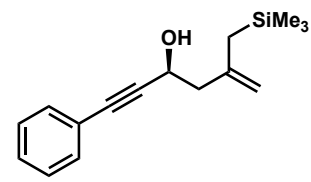

rac-1-phenyl-5-((trimethylsilyl)methyl)hex-5-en-1-yn-3-ol

(3k)

Prepared according to the general procedure. The crude mixture was purified by column chromatography to give compound $\mathbf{3 k}$ in $82 \%$ yield $(21 \mathrm{mg})$ as colorless oil. ${ }^{1} \mathrm{H}$ NMR $\left(600 \mathrm{MHz}, d^{6}\right.$-acteone) $\delta 7.38-7.54(\mathrm{~m}, 2 \mathrm{H})$, 
7.35 (app. s, 3H), 4.79 (s, 1H), $4.65-4.73(\mathrm{~m}, 2 \mathrm{H}), 4.52(\mathrm{~d}, J=5.1 \mathrm{~Hz}, 1 \mathrm{H}), 2.48$ (dd, $J$ $=13.7,6.6 \mathrm{~Hz}, 1 \mathrm{H}), 2.42(\mathrm{dd}, J=13.6,7.2 \mathrm{~Hz}, 1 \mathrm{H}), 1.72(\mathrm{~d}, J=13.4 \mathrm{~Hz}, 1 \mathrm{H}), 1.68(\mathrm{~d}, J$ $=13.4 \mathrm{~Hz}, 1 \mathrm{H}), 0.05(\mathrm{~s}, 9 \mathrm{H}) \cdot{ }^{13} \mathrm{C}$ NMR $\left(151 \mathrm{MHz}, d^{6}\right.$-acteone) $\delta 144.2,132.1,129.3$, 129.0, 124.0, 110.6, 92.5, 84.3, 61.8, 47.5, 27.3, -1.3. HRMS (EI): $\mathrm{m} / \mathrm{z}$ for $\mathrm{C}_{16} \mathrm{H}_{20} \mathrm{Si}$ [M- $\left.\mathrm{H}_{2} \mathrm{O}\right]^{+}$calcd. 240.1334 , found: 240.1326 .

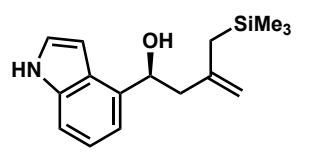

rac-1-(1H-indol-4-yl)-3-((trimethylsilyl)methyl)but-3-en-1-ol

Prepared according to the general procedure. The crude mixture was purified by column chromatography to give compound 31 in $93 \%$ yield $(25 \mathrm{mg})$ as colorless oil. ${ }^{1} \mathrm{H}$ NMR $\left(400 \mathrm{MHz}, d^{6}\right.$-acteone) $\delta 7.30-7.32(\mathrm{~m}, 2 \mathrm{H}), 7.12(\mathrm{~d}$, $J=7.1 \mathrm{~Hz}, 1 \mathrm{H}), 7.06(\mathrm{dd}, J=7.6,7.6 \mathrm{~Hz}, 1 \mathrm{H}), 6.63(\mathrm{~s}, 1 \mathrm{H}), 5.22(\mathrm{ddd}, J=8.7,4.5,4.5$ $\mathrm{Hz}, 1 \mathrm{H}), 4.75(\mathrm{~s}, 1 \mathrm{H}), 4.62(\mathrm{~s}, 1 \mathrm{H}), 3.81(\mathrm{~d}, J=3.6 \mathrm{~Hz}, 1 \mathrm{H}), 2.43-2.57$ (m, 2H), 1.73 (d, $J=13.4 \mathrm{~Hz}, 1 \mathrm{H}), 1.65(\mathrm{~d}, J=13.4 \mathrm{~Hz}, 1 \mathrm{H}), 0.03(\mathrm{~s}, 9 \mathrm{H}) .{ }^{13} \mathrm{C} \mathrm{NMR}(151 \mathrm{MHz}$, $d^{6}$-acteone) $\delta 146.1,138.3,137.2,126.2,125.0,121.9,116.3,110.8,109.9,100.8$ (100.7), 71.8 (71.7), 48.41 (48.35), 27.2, -1.2. HRMS (ESI): $\mathrm{m} / \mathrm{z}$ for $\mathrm{C}_{16} \mathrm{H}_{23} \mathrm{NOSiNa}[\mathrm{M}+\mathrm{Na}]^{+}$ calcd. 296.1447, found: 296.1443 .

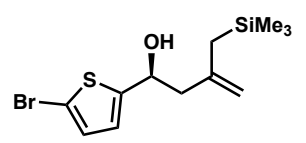

rac-1-(5-bromothiophen-2-yl)-3-((trimethylsilyl)methyl)but-3-en-1

-ol (3m) Prepared according to the general procedure. The crude mixture was purified by column chromatography to give compound $3 \mathbf{m}$ in $67 \%$ yield $(21 \mathrm{mg})$ as colorless oil. ${ }^{1} \mathrm{H}$ NMR $\left(600 \mathrm{MHz}, d^{6}\right.$-acteone) $\delta 6.93-7.00$ $(\mathrm{m}, 1 \mathrm{H}), 6.78(\mathrm{~d}, J=2.8 \mathrm{~Hz}, 1 \mathrm{H}), 4.99-5.11(\mathrm{~m}, 1 \mathrm{H}), 4.71(\mathrm{~d}, J=3.6 \mathrm{~Hz}, 1 \mathrm{H}), 4.67(\mathrm{~s}$, $1 \mathrm{H}), 4.61(\mathrm{~s}, 1 \mathrm{H}), 2.48(\mathrm{dd}, J=13.8,7.9 \mathrm{~Hz}, 1 \mathrm{H}), 2.40(\mathrm{dd}, J=13.9,5.2 \mathrm{~Hz}, 1 \mathrm{H}), 1.65$ (d, $J=13.4 \mathrm{~Hz}, 1 \mathrm{H}), 1.60(\mathrm{~d}, J=13.4 \mathrm{~Hz}, 1 \mathrm{H}), 0.03(\mathrm{~s}, 9 \mathrm{H}) .{ }^{13} \mathrm{C} \mathrm{NMR}\left(151 \mathrm{MHz}, d^{6}\right.$-acteone) $\delta 152.6,143.7,129.5,123.5,110.0,109.5,68.4$ (68.3), 48.31 (48.26), 26.3, -2.1. HRMS (ESI): $\mathrm{m} / \mathrm{z}$ for $\mathrm{C}_{12} \mathrm{H}_{18} \mathrm{BrSSi}[\mathrm{M}-\mathrm{OH}]^{+}$calcd. 301.0082, found: 301.0069 .

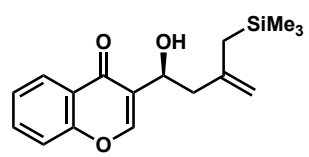

rac-3-(1-hydroxy-3-((trimethylsilyl)methyl)but-3-en-1-yl)-4H-chr omen-4-one (3n) Prepared according to the general procedure. The crude mixture was purified by column chromatography to give compound $3 \mathrm{n}$ in $95 \%$ yield $\left(29 \mathrm{mg}\right.$ ) as colorless oil. ${ }^{1} \mathrm{H}$ NMR (600 MHz, $d^{6}$-acteone) $\delta$ $8.18(\mathrm{~s}, 1 \mathrm{H}), 8.14(\mathrm{~d}, J=7.9 \mathrm{~Hz}, 1 \mathrm{H}), 7.79$ (dd, $J=7.7,7.7 \mathrm{~Hz}, 1 \mathrm{H}), 7.58$ (d, $J=8.4 \mathrm{~Hz}$, 1H), 7.48 (dd, $J=7.3,7.3 \mathrm{~Hz}, 1 \mathrm{H}), 4.96-5.02(\mathrm{~m}, 1 \mathrm{H}), 4.69(\mathrm{~s}, 1 \mathrm{H}), 4.60(\mathrm{~s}, 1 \mathrm{H}), 4.21$ $(\mathrm{d}, J=3.6 \mathrm{~Hz}, 1 \mathrm{H}), 2.61(\mathrm{~d}, J=13.8 \mathrm{~Hz}, 1 \mathrm{H}), 2.23(\mathrm{dd}, J=13.6,8.7 \mathrm{~Hz}, 1 \mathrm{H}), 1.72(\mathrm{~d}, J$ $=13.4 \mathrm{~Hz}, 1 \mathrm{H}), 1.66(\mathrm{~d}, J=13.4 \mathrm{~Hz}, 1 \mathrm{H}), 0.05(\mathrm{~s}, 9 \mathrm{H}) .{ }^{13} \mathrm{C} \mathrm{NMR}\left(151 \mathrm{MHz}, d^{6}\right.$-acteone) $\delta 176.9,157.1,154.0,145.5,134.6,128.0,126.1,125.9,124.7,119.1,110.4$ (110.3), 66.1 
(66.0), 46.4 (46.3), 27.0, -1.3. HRMS (ESI): $\mathrm{m} / \mathrm{z}$ for $\mathrm{C}_{17} \mathrm{H}_{22} \mathrm{O}_{3} \mathrm{SiNa}[\mathrm{M}+\mathrm{Na}]^{+}$calcd. 325.1236, found: 325.1222 .

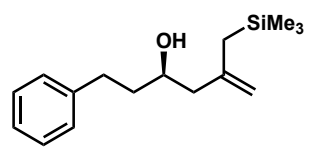

rac-1-phenyl-5-((trimethylsilyl)methyl)hex-5-en-3-ol (3o) Prepared according to the general procedure. The crude mixture was purified by column chromatography to give compound 30 in $86 \%$ yield ( 22 $\mathrm{mg})$ as colorless oil. ${ }^{1} \mathrm{H} \mathrm{NMR}\left(600 \mathrm{MHz}, d^{6}\right.$-acteone) $\delta 7.26(\mathrm{dd}, J=7.1,7.1 \mathrm{~Hz}, 2 \mathrm{H})$, $7.21(\mathrm{~d}, J=7.0 \mathrm{~Hz}, 2 \mathrm{H}), 7.10-7.18(\mathrm{~m}, 1 \mathrm{H}), 4.63(\mathrm{~s}, 1 \mathrm{H}), 4.57(\mathrm{~s}, 1 \mathrm{H}), 3.75(\mathrm{~s}, 1 \mathrm{H})$, $3.45(\mathrm{~s}, 1 \mathrm{H}), 2.75-2.87(\mathrm{~m}, 1 \mathrm{H}), 2.60-2.71(\mathrm{~m}, 1 \mathrm{H}), 2.10-2.25(\mathrm{~m}, 2 \mathrm{H}), 1.71-1.88$ $(\mathrm{m}, 1 \mathrm{H}), 1.59-1.70(\mathrm{~m}, 1 \mathrm{H}), 1.57(\mathrm{~s}, 2 \mathrm{H}), 0.01(\mathrm{~s}, 9 \mathrm{H}) .{ }^{13} \mathrm{C}$ NMR (151 MHz, $d^{6}$-acteone) $\delta 145.9,143.6,129.2,129.1,126.4,109.84$ (109.82), 69.1 (69.0), 47.53 (47.48), 40.0, (39.9), 32.7, 27.1, -1.3. HRMS (ESI): $\mathrm{m} / \mathrm{z}$ for $\mathrm{C}_{16} \mathrm{H}_{26} \mathrm{OSiNa}[\mathrm{M}+\mathrm{Na}]^{+}$calcd. 285.1651, found: 285.1647.

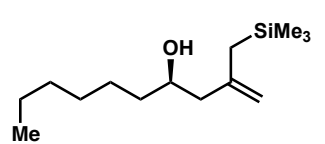

rac-2-(trimethylsilyl)methyl)dec-1-en-4-ol (3p) Prepared according to the general procedure. The crude mixture was purified by column chromatography to give compound $\mathbf{3 p}$ in $97 \%$ yield $(23 \mathrm{mg})$ as colorless oil. ${ }^{1} \mathrm{H}$ NMR (600 MHz, $d^{6}$-acteone) $\delta 4.63(\mathrm{~s}, 1 \mathrm{H}), 4.57(\mathrm{~s}, 1 \mathrm{H}), 3.70(\mathrm{bs}, 1 \mathrm{H})$, $2.10(\mathrm{~d}, J=6.2 \mathrm{~Hz}, 2 \mathrm{H}), 1.60(\mathrm{~s}, 2 \mathrm{H}), 1.47(\mathrm{~d}, J=11.8 \mathrm{~Hz}, 2 \mathrm{H}), 1.22-1.38(\mathrm{~m}, 8 \mathrm{H})$, $0.87(\mathrm{~d}, J=6.9 \mathrm{~Hz}, 3 \mathrm{H}), 0.02(\mathrm{~s}, 9 \mathrm{H}) .{ }^{13} \mathrm{C}$ NMR $\left(151 \mathrm{MHz}, d^{6}\right.$-acteone) $\delta 146.1,109.71$ (109.69), 69.6 (69.5), 47.6 (47.5), 38.02 (37.97), 32.7, 30.2, 27.1, 26.5, 23.3, 14.4, -1.3. HRMS (ESI): $\mathrm{m} / \mathrm{z}$ for $\mathrm{C}_{14} \mathrm{H}_{30} \mathrm{OSiNa}[\mathrm{M}+\mathrm{Na}]^{+}$calcd. 265.1964, found: 265.1958.

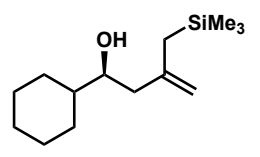

rac-1-cyclohexyl-3-((trimethylsilyl)methyl)but-3-en-1-ol (3q) Prepared according to the general procedure. The crude mixture was purified by column chromatography to give compound $\mathbf{3 q}$ in $94 \%$ yield $(23 \mathrm{mg})$ as colorless oil. ${ }^{1} \mathrm{H}$ NMR (400 MHz, $d^{6}$-acteone) $\delta 4.66(\mathrm{~s}, 1 \mathrm{H}), 4.60(\mathrm{~s}, 1 \mathrm{H}), 3.40-3.58(\mathrm{~m}$, 1H), $2.94(\mathrm{~d}, J=4.6 \mathrm{~Hz}, 1 \mathrm{H}), 2.20(\mathrm{dd}, J=13.8,3.4 \mathrm{~Hz}, 1 \mathrm{H}), 1.91-2.03(\mathrm{~m}, 1 \mathrm{H}), 1.84$ $(\mathrm{d}, J=11.3 \mathrm{~Hz}, 1 \mathrm{H}), 1.69-1.78(\mathrm{~m}, 2 \mathrm{H}), 1.55-1.68(\mathrm{~m}, 4 \mathrm{H}), 0.85-1.48(\mathrm{~m}, 6 \mathrm{H}), 0.03$ (s, 9H). ${ }^{13} \mathrm{C}$ NMR (151 MHz, $d^{6}$-acteone) $\delta$ 146.4, 109.80 (109.78), 73.5 (73.4), 44.34 (44.31), 44.01 (43.96), 28.2, 27.3, 27.2, 27.0, 26.9, 25.1, -1.2. HRMS (CI+): m/z for $\mathrm{C}_{14} \mathrm{H}_{27} \mathrm{OSi}[\mathrm{M}-\mathrm{H}]^{+}$calcd. 239.1831, found: 239.1824 .

$\overbrace{\text { according to the general procedure. The crude mixture was purified by }}^{\text {me }}$ column chromatography to give compound $3 \mathbf{r}$ in $67 \%$ yield $(14 \mathrm{mg})$ as colorless oil. ${ }^{1} \mathrm{H}$ 
NMR (600 MHz, $d^{6}$-acteone) $\delta 4.63(\mathrm{~s}, 1 \mathrm{H}), 4.58(\mathrm{~s}, 1 \mathrm{H}), 3.80(\mathrm{bs}, 1 \mathrm{H}), 3.21(\mathrm{~s}, 1 \mathrm{H})$, $2.06-2.20(\mathrm{~m}, 2 \mathrm{H}), 1.77-1.92(\mathrm{~m}, 1 \mathrm{H}), 1.60(\mathrm{~s}, 2 \mathrm{H}), 1.26-1.40(\mathrm{~m}, 1 \mathrm{H}), 1.12-1.26$ $(\mathrm{m}, 1 \mathrm{H}), 0.89(\mathrm{~d}, J=6.4, \mathrm{~Hz}, 3 \mathrm{H}), 0.88(\mathrm{~d}, J=6.4, \mathrm{~Hz}, 3 \mathrm{H}), 0.03(\mathrm{~s}, 9 \mathrm{H}) .{ }^{13} \mathrm{C} \mathrm{NMR}(151$ $\mathrm{MHz}, d^{6}$-acteone) $\delta 146.0,109.76$ (109.74), 67.6 (67.5), 48.2 (48.1), 47.3 (47.2), 27.1, 25.1, 24.0, 22.1, -1.3. HRMS (CI+): $\mathrm{m} / \mathrm{z}$ for $\mathrm{C}_{12} \mathrm{H}_{25} \mathrm{OSi}[\mathrm{M}-\mathrm{H}]^{+}$calcd. 213.1675, found: 213.1672 .

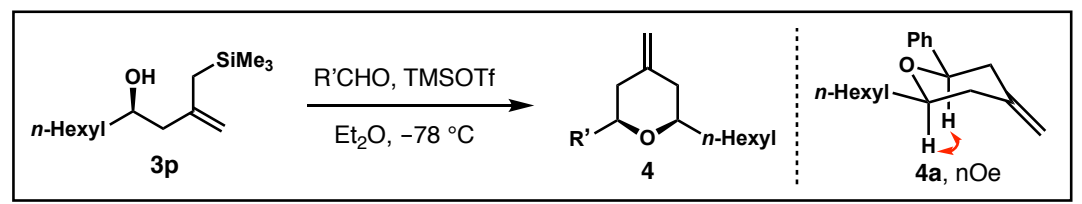

General procedure for stereoselective synthesis of tetrahydropyrans 4: To a solution of alcohol 3p (24 mg, $0.1 \mathrm{mmol}, 1.0$ equiv) in diethyl ether $(2.0 \mathrm{~mL})$, aldehyde $(0.2 \mathrm{mmol}$, 2.0 equiv) was added. The reaction mixture was placed in a dry ice/acetone bath and cooled to $-78{ }^{\circ} \mathrm{C}$. TMSOTf ( $27 \mu \mathrm{L}, 0.15 \mathrm{mmol}, 1.5$ equiv) was added and the mixture was stirred at $-78{ }^{\circ} \mathrm{C}$ for $20 \mathrm{~min}$. Then aqueous $\mathrm{NaOH}$ solution $(1 \mathrm{~mL}, 1 \mathrm{~N})$ was added and the mixture was allowed to warm to ambient temperature. Then the reaction mixture was transferred into a separatory funnel. The organic layer was separated and the aqueous layer was extracted with $\mathrm{Et}_{2} \mathrm{O}(3 \times 1 \mathrm{~mL})$. The combined organic layers were dried over anhydrous magnesium sulfate, filtered, and concentrated under reduced pressure. Purification of the crude product was performed by flash chromatography (gradient elution with hexane and EtOAc) to provide product 4.

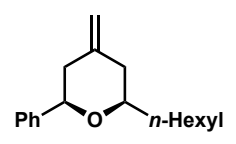

rac-(2R,6R)-2-hexyl-4-methylene-6-phenyltetrahydro-2H-pyran

(4a)

Prepared according to the general procedure. The crude mixture was purified by column chromatography to give compound $\mathbf{4 a}$ in $73 \%$ yield $(19 \mathrm{mg})$ as colorless oil. ${ }^{1} \mathrm{H}$ NMR $\left(600 \mathrm{MHz}, \mathrm{CDCl}_{3}\right) \delta 7.39(\mathrm{~d}, J=7.1 \mathrm{~Hz}, 2 \mathrm{H}), 7.35$ (dd, $J=7.4,7.4 \mathrm{~Hz}, 2 \mathrm{H}), 7.19-7.30(\mathrm{~m}, 1 \mathrm{H}), 4.79$ (s, 2H), $4.23-4.40(\mathrm{~m}, 1 \mathrm{H}), 3.24-3.60$ (m, 1H), 2.46 (d, $J=13.3 \mathrm{~Hz}, 1 \mathrm{H}), 2.29$ (d, $J=13.2 \mathrm{~Hz}, 1 \mathrm{H}), 2.22(\mathrm{dd}, J=12.1 \mathrm{~Hz}, 12.1$ $\mathrm{Hz}, 1 \mathrm{H}), 2.02(\mathrm{dd}, J=12.1 \mathrm{~Hz}, 12.1 \mathrm{~Hz}, 1 \mathrm{H}), 1.63-1.78(\mathrm{~m}, 1 \mathrm{H}), 1.49-1.56(\mathrm{~m}, 1 \mathrm{H})$, $1.41-1.49(\mathrm{~m}, 1 \mathrm{H}), 1.34-1.41(\mathrm{~m}, 1 \mathrm{H}), 1.09-1.34(\mathrm{~m}, 6 \mathrm{H}), 0.76-0.99(\mathrm{~m}, 3 \mathrm{H}) .{ }^{13} \mathrm{C}$ NMR $\left(151 \mathrm{MHz}, \mathrm{CDCl}_{3}\right) \delta 145.4,143.0,128.7,127.7,126.2,109.0,80.3,79.2,43.1$, 40.8, 36.7, 32.2, 29.7, 25.7, 23.0, 14.5. HRMS (ESI): $\mathrm{m} / \mathrm{z}$ for $\mathrm{C}_{18} \mathrm{H}_{27} \mathrm{O}[\mathrm{M}+\mathrm{H}]^{+}$calcd. 259.2062, found: 259.2051. 


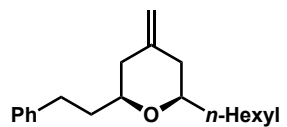

rac-(2R,6S)-2-hexyl-4-methylene-6-phenethyltetrahydro-2H-pyran

(4b) Prepared according to the general procedure. The crude mixture was purified by column chromatography to give compound $\mathbf{4 b}$ in $70 \%$ yield $(20 \mathrm{mg})$ as colorless oil. ${ }^{1} \mathrm{H}$ NMR $\left(600 \mathrm{MHz}, \mathrm{CDCl}_{3}\right) \delta 7.23-7.39(\mathrm{~m}, 2 \mathrm{H}), 7.18-$ $7.22(\mathrm{~m}, 3 \mathrm{H}), 4.68(\mathrm{~s}, 1 \mathrm{H}), 4.66(\mathrm{~s}, 1 \mathrm{H}), 3.03-3.34(\mathrm{~m}, 2 \mathrm{H}), 2.76-2.92(\mathrm{~m}, 1 \mathrm{H}), 2.61-$ $2.76(\mathrm{~m}, 1 \mathrm{H}), 2.20(\mathrm{~d}, J=13.4 \mathrm{~Hz}, 1 \mathrm{H}), 2.17(\mathrm{~d}, J=13.6 \mathrm{~Hz}, 1 \mathrm{H}), 1.81-2.03(\mathrm{~m}, 3 \mathrm{H})$, $1.66-1.79(\mathrm{~m}, 1 \mathrm{H}), 1.07-1.65(\mathrm{~m}, 10 \mathrm{H}), 0.76-1.00(\mathrm{~m}, 3 \mathrm{H}) .{ }^{13} \mathrm{C}$ NMR $(151 \mathrm{MHz}$, $\left.\mathrm{CDCl}_{3}\right) \delta 145.5,142.5,128.9,128.6,126.0,108.5,78.6,77.3,41.4,41.3,38.2,36.7,32.2$, 32.1, 29.7, 26.0, 23.0, 14.5. HRMS (ESI): $\mathrm{m} / \mathrm{z}$ for $\mathrm{C}_{20} \mathrm{H}_{31} \mathrm{O}[\mathrm{M}+\mathrm{H}]^{+}$calcd. 287.2375, found: 287.2361 .

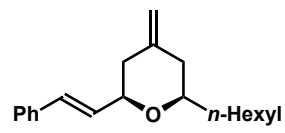

rac-(2R,6R)-2-hexyl-4-methylene-6-((E)-styryl)tetrahydro-2H-pyra n (4c) Prepared according to the general procedure. The crude mixture was purified by column chromatography to give compound $4 \mathbf{c}$ in $96 \%$ yield $(27 \mathrm{mg})$ as colorless oil. ${ }^{1} \mathrm{H}$ NMR $\left(600 \mathrm{MHz}, \mathrm{CDCl}_{3}\right) \delta 7.39(\mathrm{~d}, J=7.2 \mathrm{~Hz}, 2 \mathrm{H})$, 7.31 (dd, $J=7.2 \mathrm{~Hz}, 7.2 \mathrm{~Hz}, 2 \mathrm{H}), 7.23$ (dd, $J=6.5 \mathrm{~Hz}, 6.5 \mathrm{~Hz}, 1 \mathrm{H}), 6.61$ (d, $J=16.0 \mathrm{~Hz}$, 1H), $6.27(\mathrm{dd}, J=16.0,5.8 \mathrm{~Hz}, 1 \mathrm{H}), 4.77(\mathrm{~s}, 2 \mathrm{H}), 3.85-4.04(\mathrm{~m}, 1 \mathrm{H}), 3.23-3.45(\mathrm{~m}$, $1 \mathrm{H}), 2.34(\mathrm{~d}, J=13.2 \mathrm{~Hz}, 1 \mathrm{H}), 2.26(\mathrm{~d}, J=13.3 \mathrm{~Hz}, 1 \mathrm{H}), 2.15(\mathrm{dd}, J=12.2 \mathrm{~Hz}, 12.2 \mathrm{~Hz}$, 1H), $1.96(\mathrm{dd}, J=12.2 \mathrm{~Hz}, 12.2 \mathrm{~Hz}, 1 \mathrm{H}), 1.61-1.72(\mathrm{~m}, 1 \mathrm{H}), 1.41-1.55(\mathrm{~m}, 2 \mathrm{H}), 1.13$ - $1.40(\mathrm{~m}, 7 \mathrm{H}), 0.78-0.97(\mathrm{~m}, 3 \mathrm{H}) .{ }^{13} \mathrm{C} \mathrm{NMR}\left(151 \mathrm{MHz}, \mathrm{CDCl}_{3}\right) \delta 144.8,137.1,130.7$, 130.4, 128.8, 127.9, 126.8, 109.1, 79.1, 78.8, 41.3, 40.8, 36.7, 32.1, 29.7, 25.8, 23.0, 14.5. HRMS (ESI): $\mathrm{m} / \mathrm{z}$ for $\mathrm{C}_{20} \mathrm{H}_{29} \mathrm{O}$ [M+H] $]^{+}$calcd. 285.2218, found: 285.2207 .
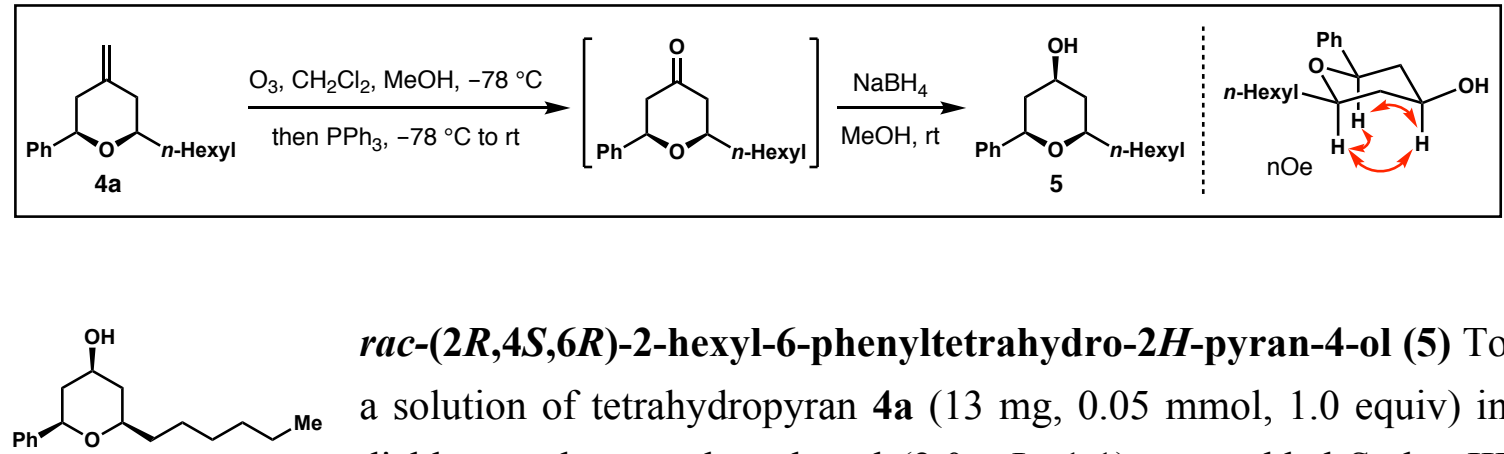

rac-(2R,4S,6R)-2-hexyl-6-phenyltetrahydro-2H-pyran-4-ol (5) To a solution of tetrahydropyran $4 \mathbf{a}(13 \mathrm{mg}, 0.05 \mathrm{mmol}, 1.0$ equiv) in dichloromethane and methanol $(2.0 \mathrm{~mL}, 1: 1)$, was added Sudan III $(1 \mathrm{mg})$ as the indicator. The reaction vessel was placed in a dry ice/acetone bath and cooled to $-78{ }^{\circ} \mathrm{C}$. Then the reaction mixture was treated with a stream of ozone (generated from air) until the bright red solution became colorless. The reaction mixture 
was purged with $\mathrm{N}_{2}$ for $5 \mathrm{~min}$, and $\mathrm{PPh}_{3}(18 \mathrm{mg}, 0.07 \mathrm{mmol}, 1.4$ equiv) was added. The reaction mixture was warmed to ambient temperature and stirred for additional $30 \mathrm{~min}$. The resulting solution was concentrated under reduced pressure. The residue was dissolved in $2 \mathrm{~mL}$ EtOH and $\mathrm{NaBH}_{4}(5.7 \mathrm{mg}, 3.0$ equiv, $0.15 \mathrm{mmol})$ was slowly added to the solution. After complete consumption of the ketone intermediate, the reaction was quenched with saturated $\mathrm{NH}_{4} \mathrm{Cl}$ solution $(3 \mathrm{~mL})$. The mixture was transferred into a separatory funnel, the organic layer was separated and the aqueous layer was extracted with EtOAc $(3 \times 1 \mathrm{~mL})$. The combined organic layers were dried over anhydrous magnesium sulfate, filtered, and concentrated under reduced pressure. Purification of the crude product was performed by flash chromatography (gradient elution with hexane and EtOAc) to provide product 5 in $98 \%$ yield $(13 \mathrm{mg})$ as colorless oil ${ }^{1} \mathrm{H}$ NMR $(600 \mathrm{MHz}$, $\left.\mathrm{CDCl}_{3}\right) \delta 7.30-7.43(\mathrm{~m}, 3 \mathrm{H}), 7.20-7.30(\mathrm{~m}, 2 \mathrm{H}), 4.36(\mathrm{~d}, J=10.7 \mathrm{~Hz}, 1 \mathrm{H}), 3.84-4.08$ (m, 1H), $3.40-3.56(\mathrm{~m}, 1 \mathrm{H}), 2.22(\mathrm{~d}, J=10.7 \mathrm{~Hz}, 1 \mathrm{H}), 2.03$ (d, $J=11.9 \mathrm{~Hz}, 1 \mathrm{H}), 1.60-$ $1.74(\mathrm{~m}, 1 \mathrm{H}), 1.13-1.60(\mathrm{~m}, 12 \mathrm{H}), 0.76-0.98(\mathrm{~m}, 3 \mathrm{H}) .{ }^{13} \mathrm{C} \mathrm{NMR}\left(151 \mathrm{MHz}, \mathrm{CDCl}_{3}\right) \delta$ $142.5,128.7,127.8,126.2,77.6,76.3,69.0,43.2$, 41.1, 36.4, 32.1, 29.7, 25.8, 23.0, 14.5. HRMS (ESI): $\mathrm{m} / \mathrm{z}$ for $\mathrm{C}_{17} \mathrm{H}_{27} \mathrm{O}_{2}[\mathrm{M}+\mathrm{H}]^{+}$calcd. 263.2011, found: 263.2007.

\section{Stability Studies of Allylboronate 2 at $-20{ }^{\circ} \mathrm{C}$}

Two samples of allylboronate $2(0.1 \mathrm{mmol})$ were prepared according to the general procedure. 1,3,5-trimethoxybenzene was used as the internal standard. ${ }^{1} \mathrm{H}$ NMR spectra were recorded in toluene- $d^{8}$ nine times over the course of two weeks. The percentage is the average of two runs.

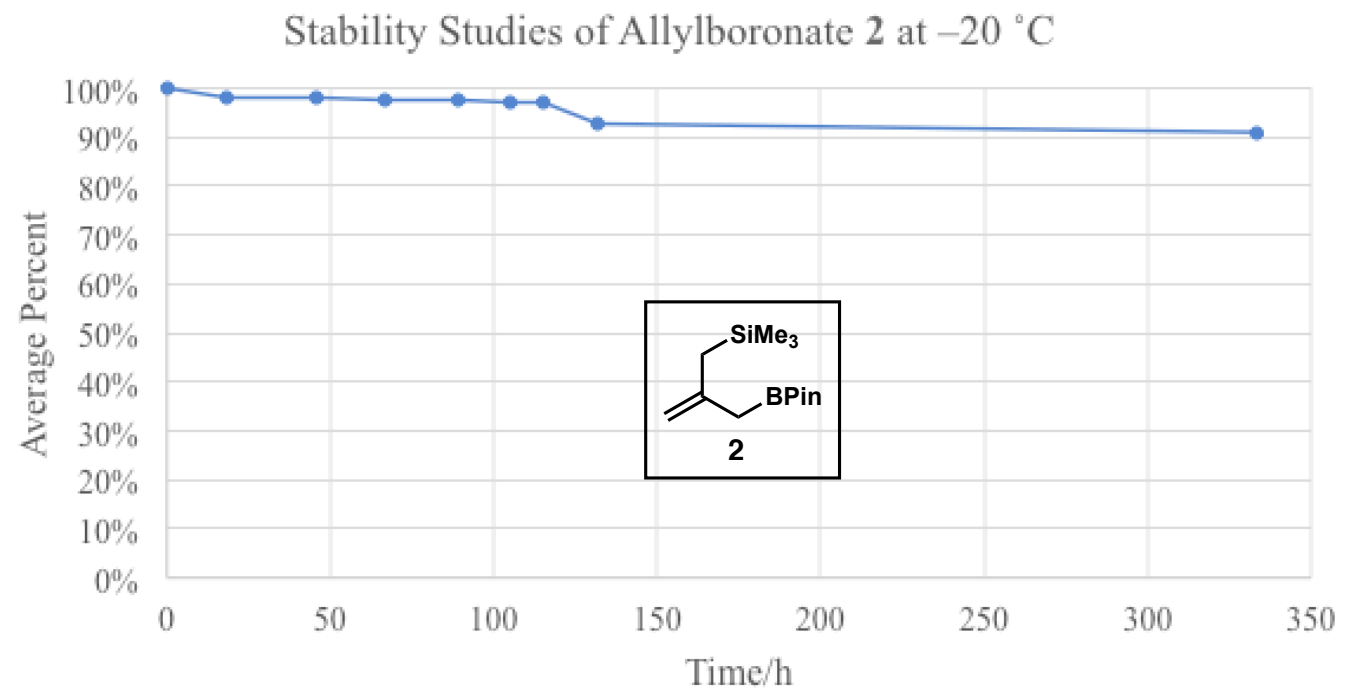



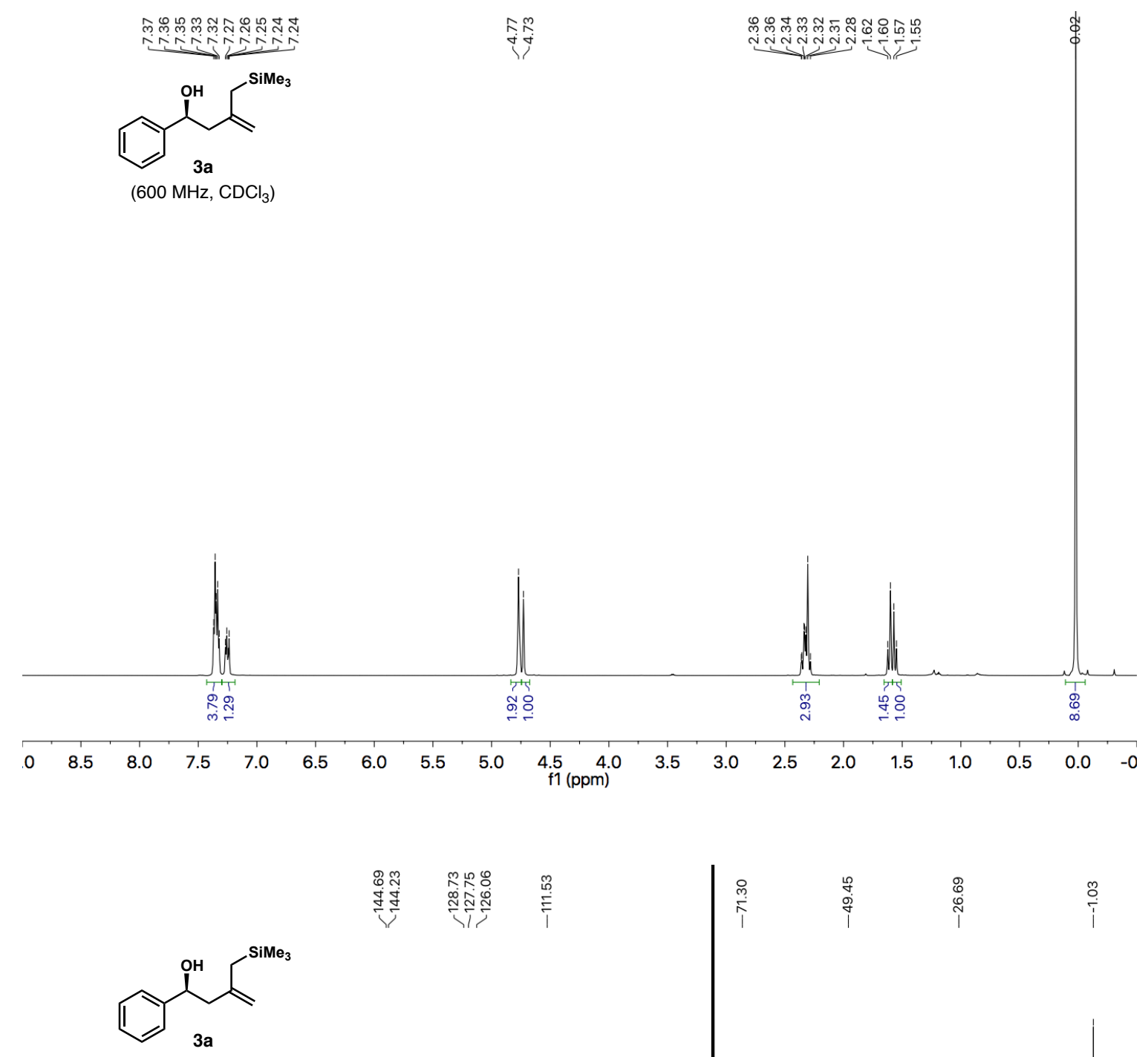

$\left(151 \mathrm{MHz}, \mathrm{CDCl}_{3}\right.$ )

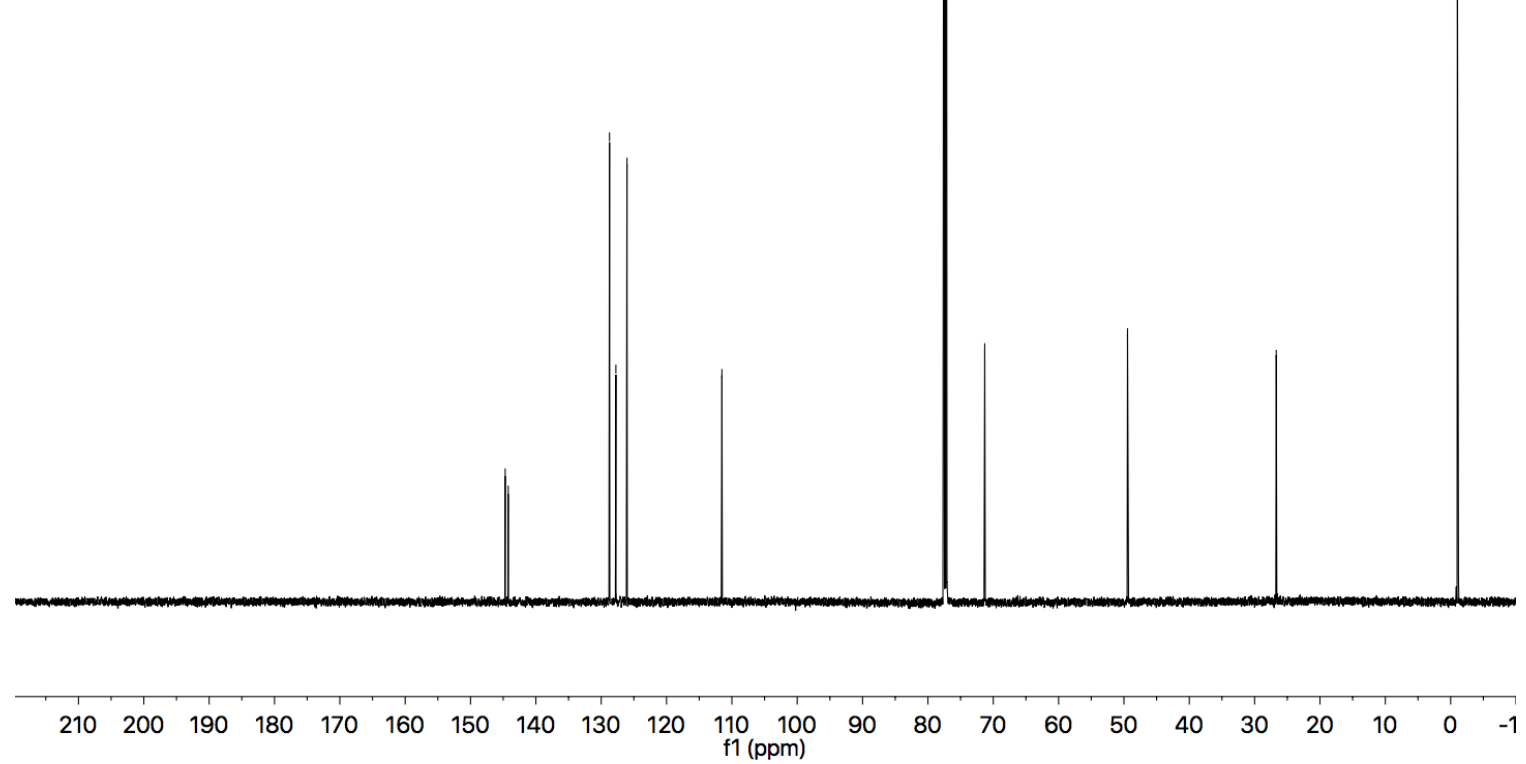

SI-12 

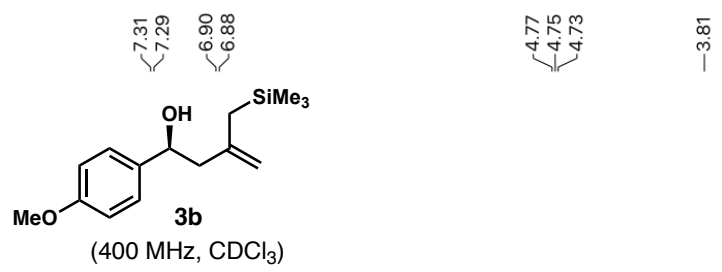

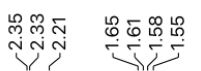

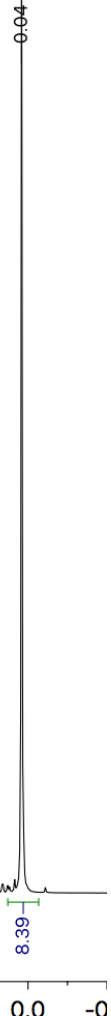

\begin{tabular}{lllllll}
\hline 0 & 8.5 & 8.0 & 7.5 & 7.0 & 6.5
\end{tabular}

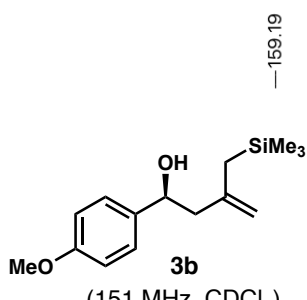

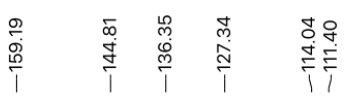

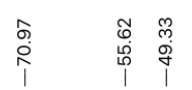

$\underset{\substack{1 \\ \stackrel{N}{N}}}{\stackrel{1}{N}}$

$\stackrel{\infty}{i}$

(151 MHz, $\mathrm{CDCl}_{3}$ )

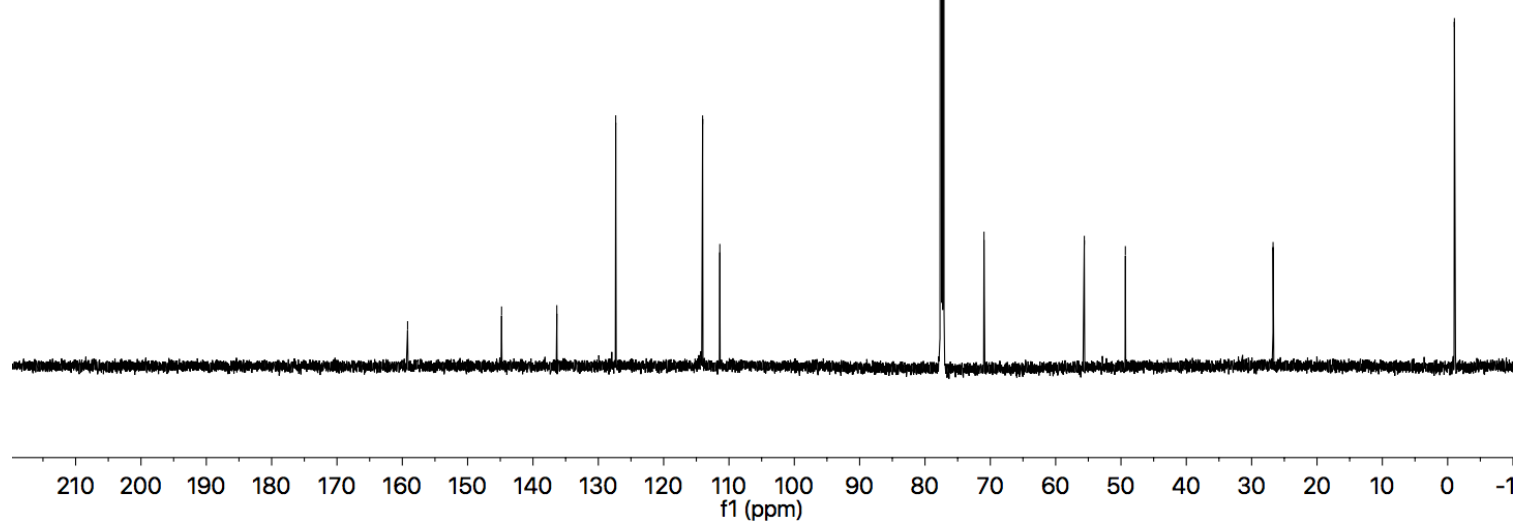

SI-13 

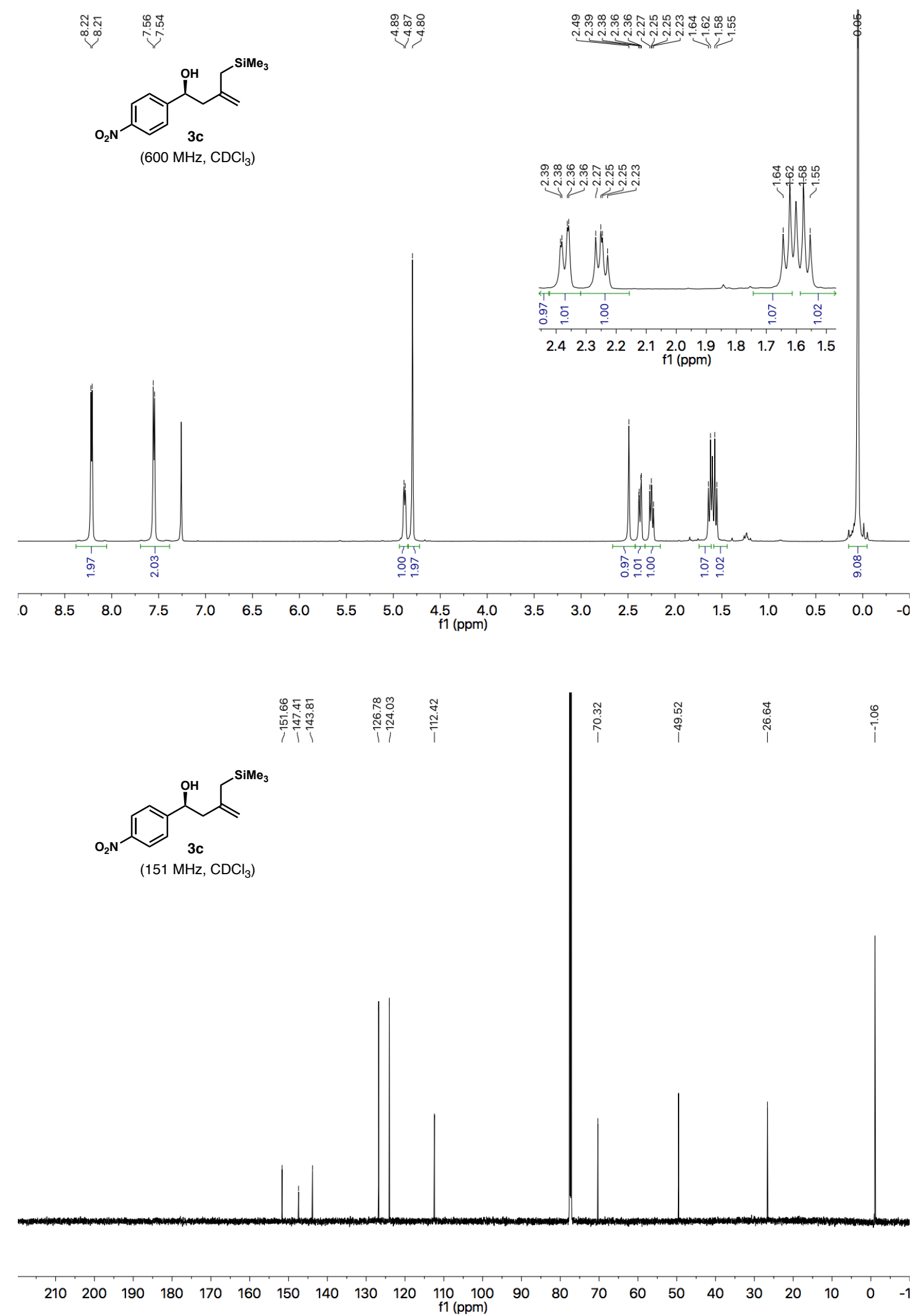

SI-14 

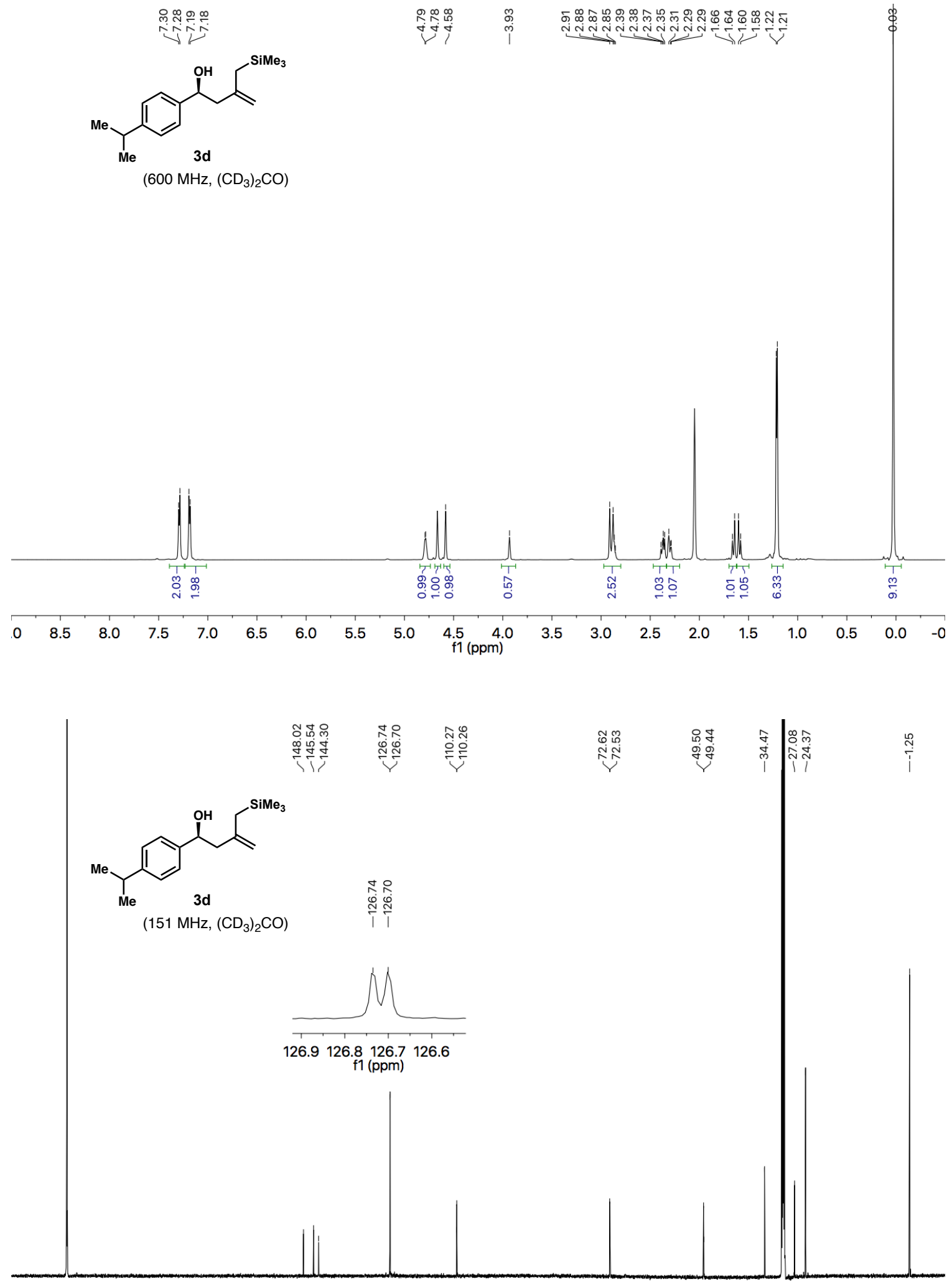

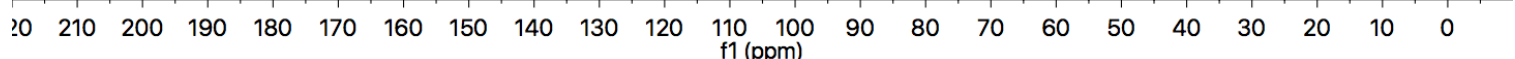

SI-15 

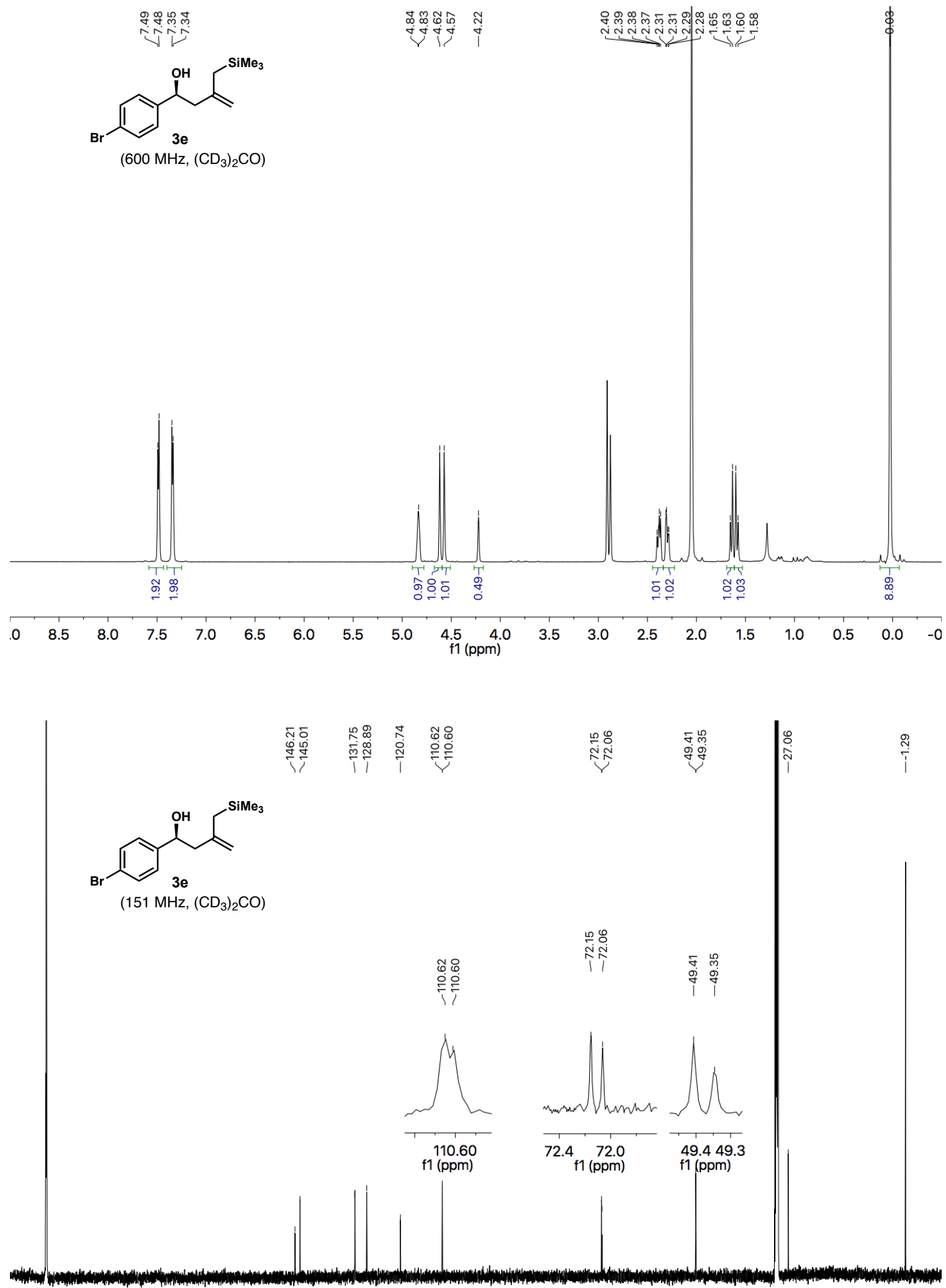

$\begin{array}{llllllllllllllllllllllll}210 & 200 & 190 & 180 & 170 & 160 & 150 & 140 & 130 & 120 & 110 & 100 & 90 & 80 & 70 & 60 & 50 & 40 & 30 & 20 & 10 & 0 & -1\end{array}$ 

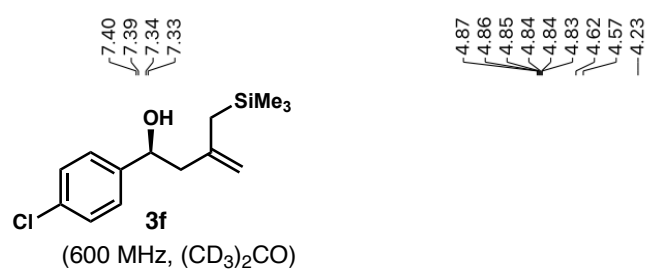

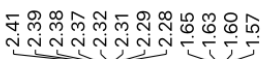

(1)

(600 $\left.\mathrm{MHZ},\left(\mathrm{CD}_{3}\right)_{2} \mathrm{CO}\right)$
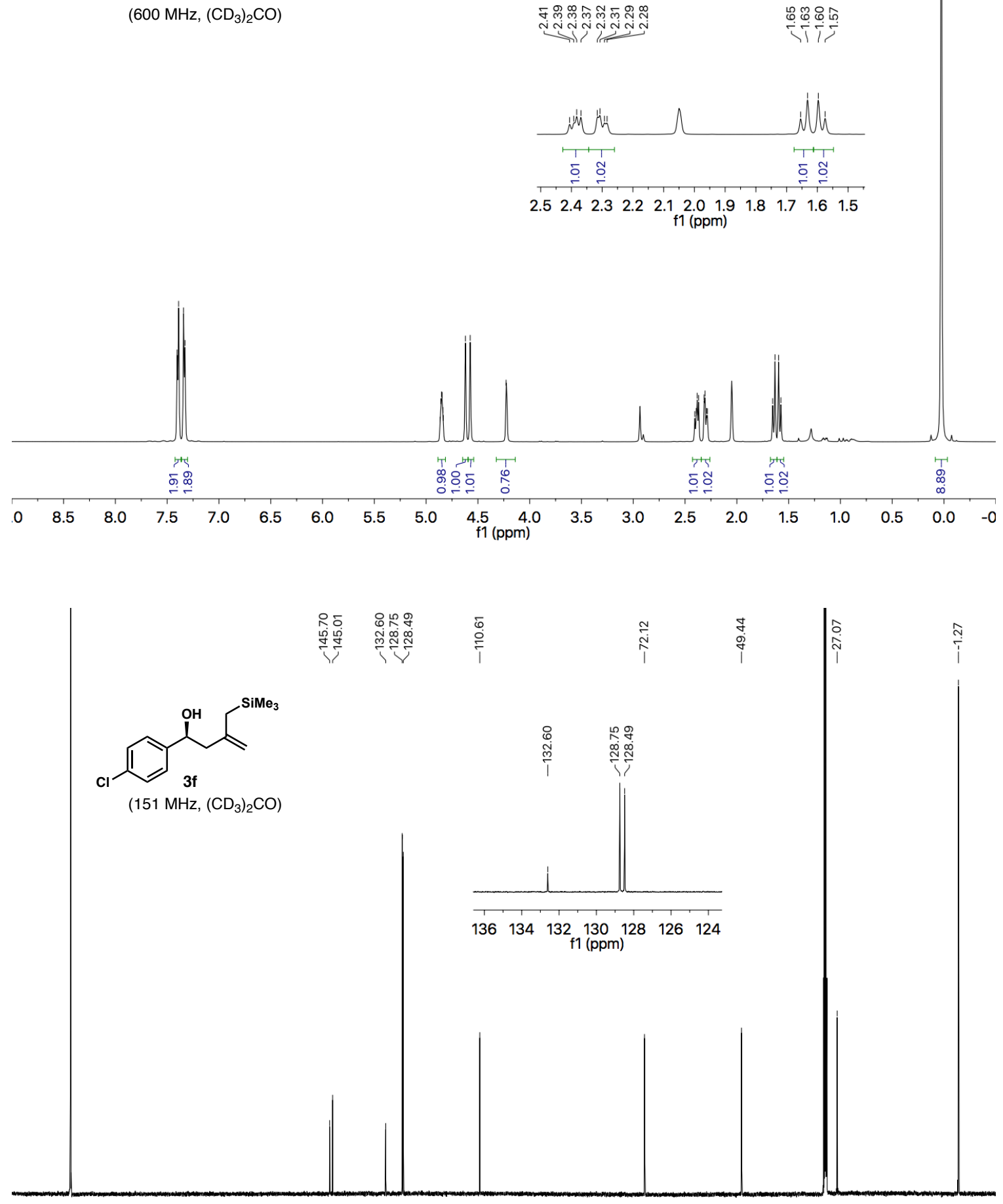

$\begin{array}{lllllllllllllllllllllllllll}.0 & 210 & 200 & 190 & 180 & 170 & 160 & 150 & 140 & 130 & 120 & 110 & 100 & 90 & 80 & 70 & 60 & 50 & 40 & 30 & 20 & 10 & 0 & -1\end{array}$ 

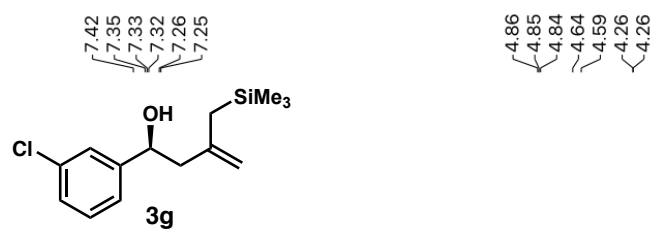

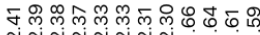

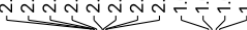

$\left(600 \mathrm{MHz},\left(\mathrm{CD}_{3}\right)_{2} \mathrm{CO}\right)$
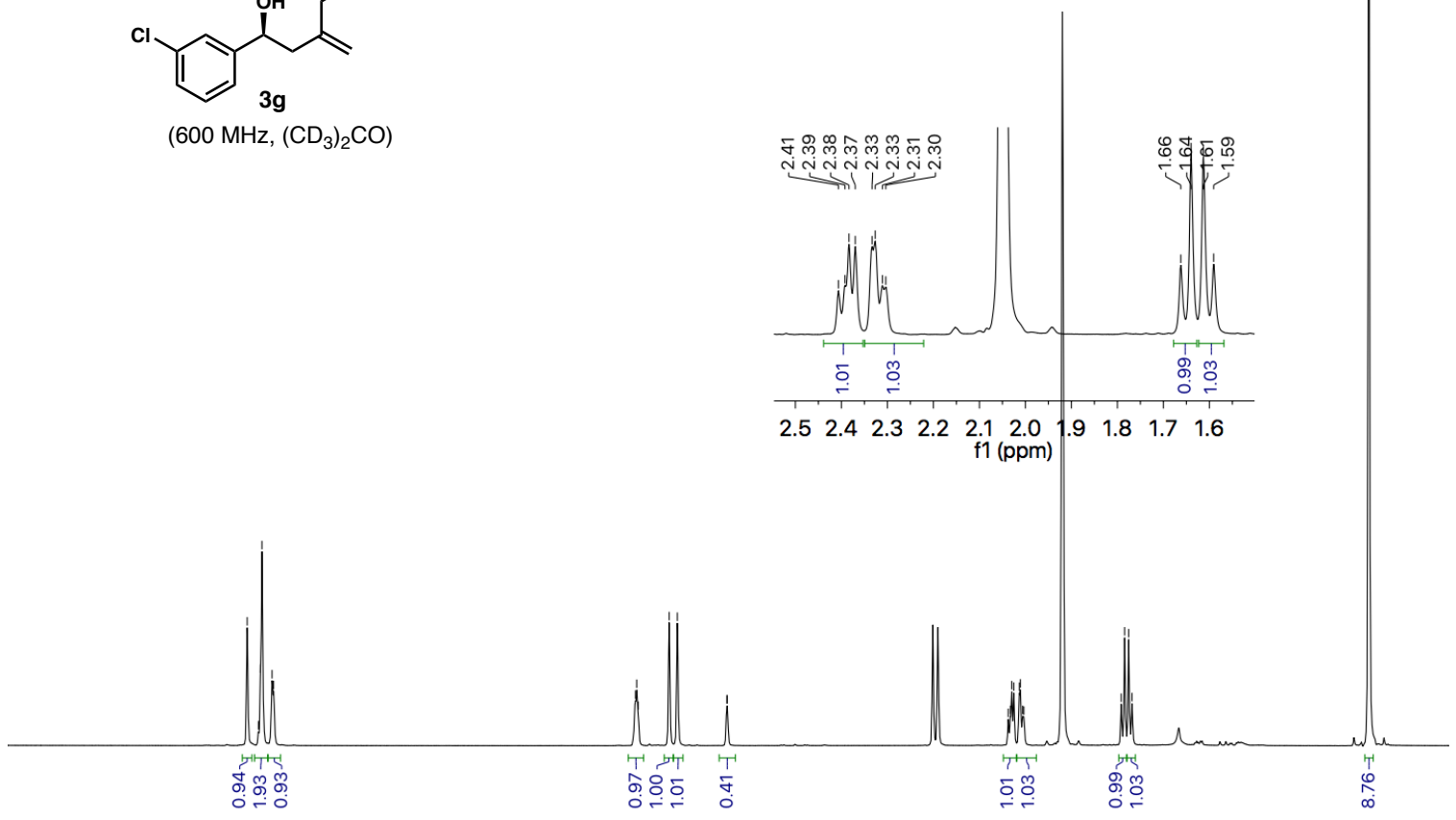

$\begin{array}{llllll}0 & 8.5 & 8.0 & 7.5 & 7.0 & 6.5\end{array}$
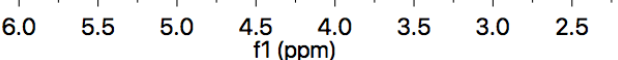

2.0
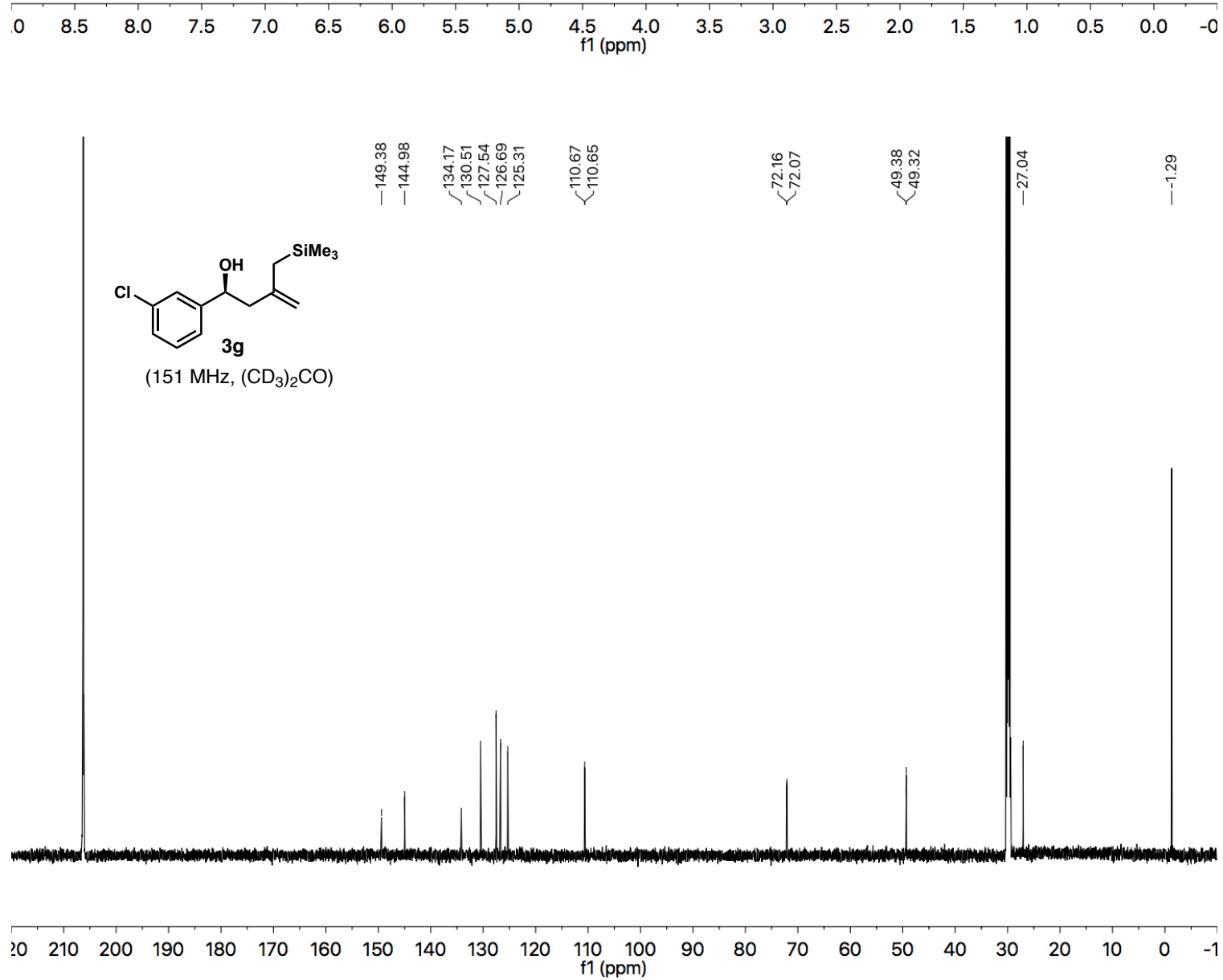

SI-18 


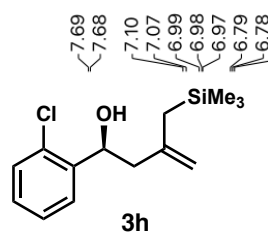

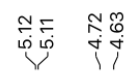

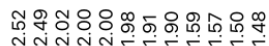

$\left(600 \mathrm{MHz}\right.$, Toluene- $\left.d_{8}\right)$
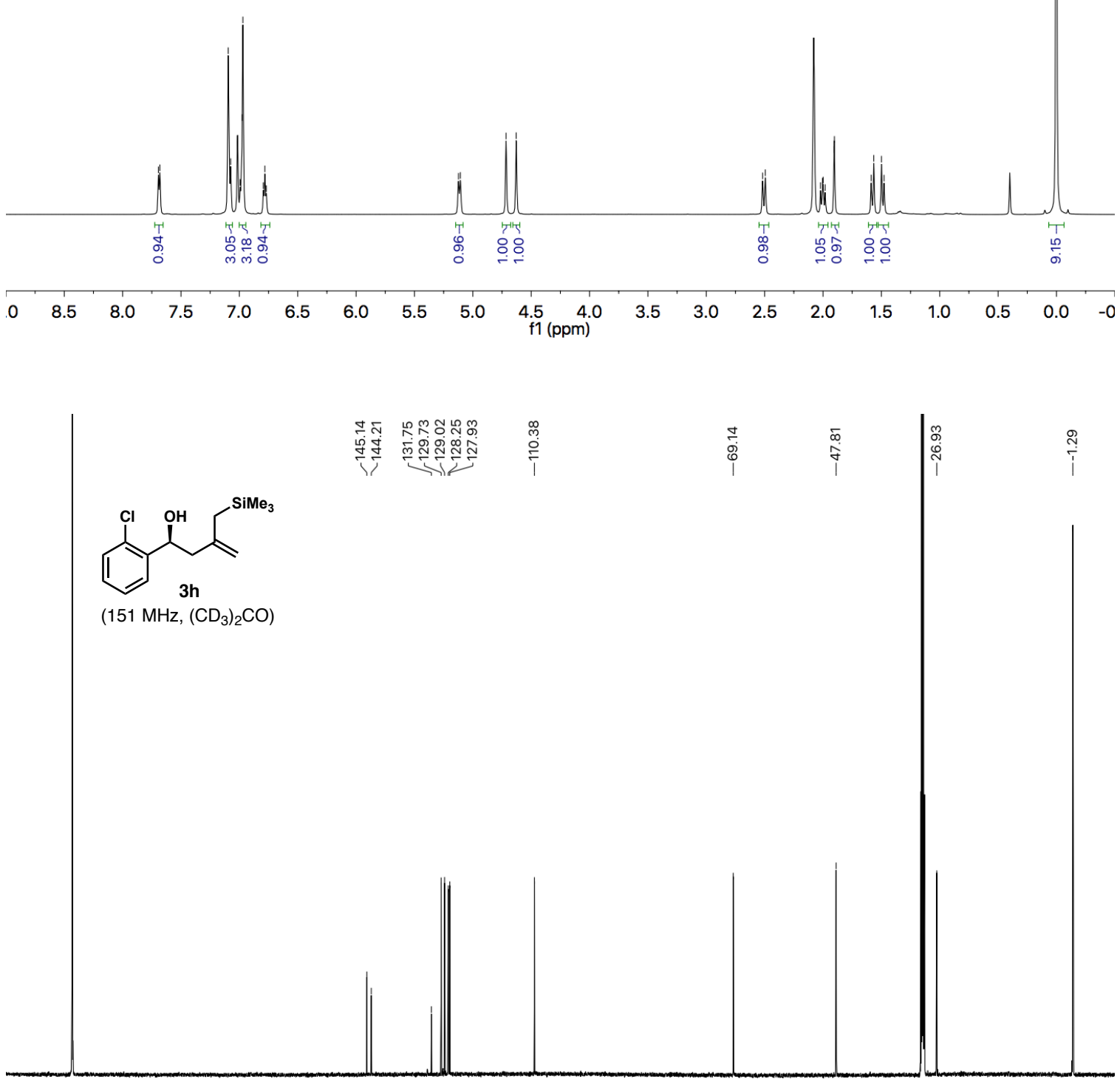

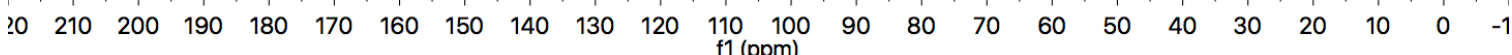

SI-19 

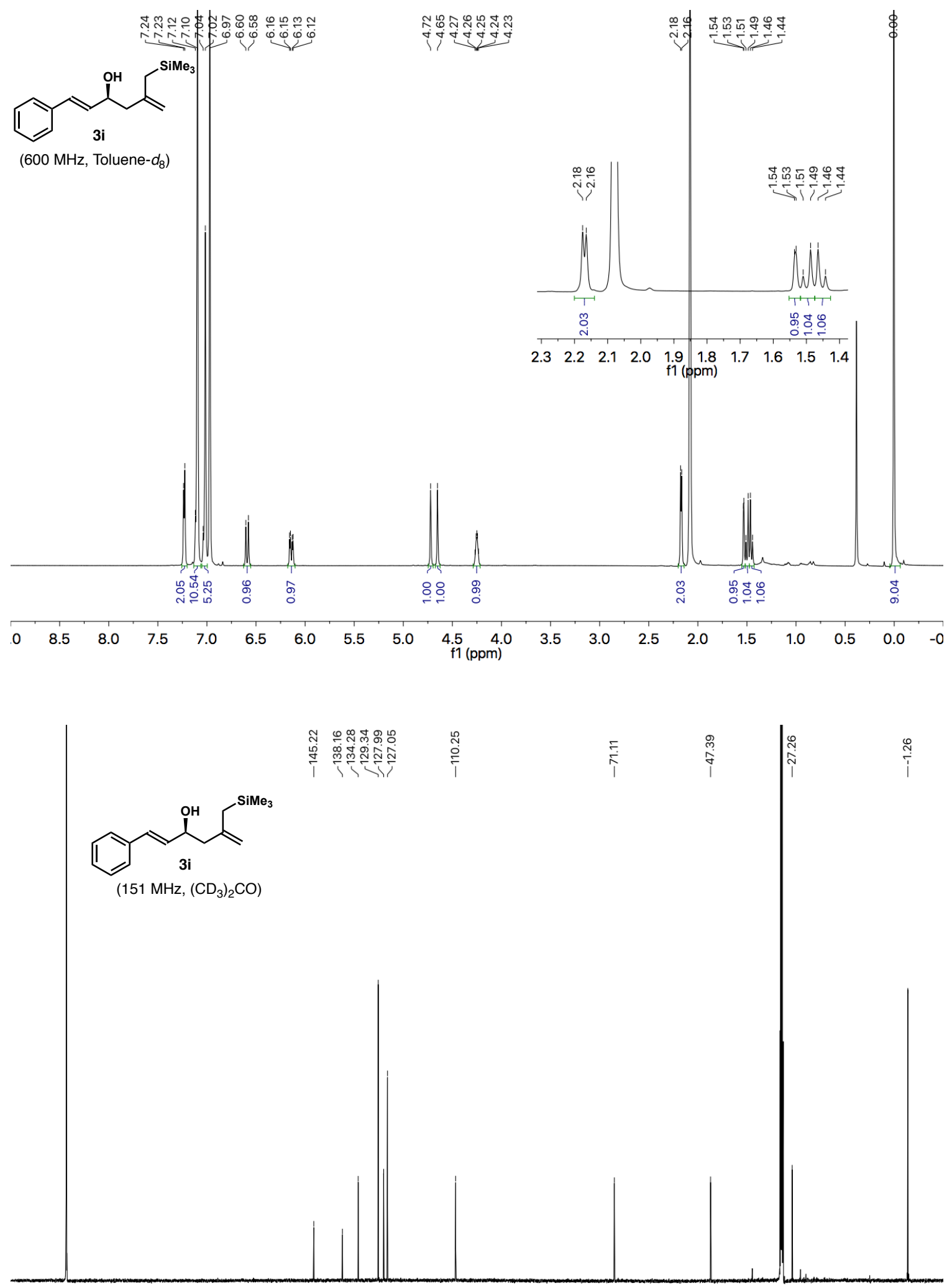

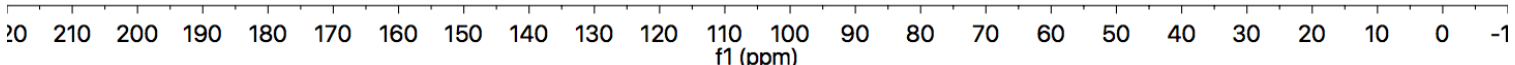




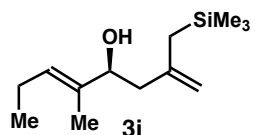

$\left(400 \mathrm{MHz},\left(\mathrm{CD}_{3}\right)_{2} \mathrm{CO}\right)$
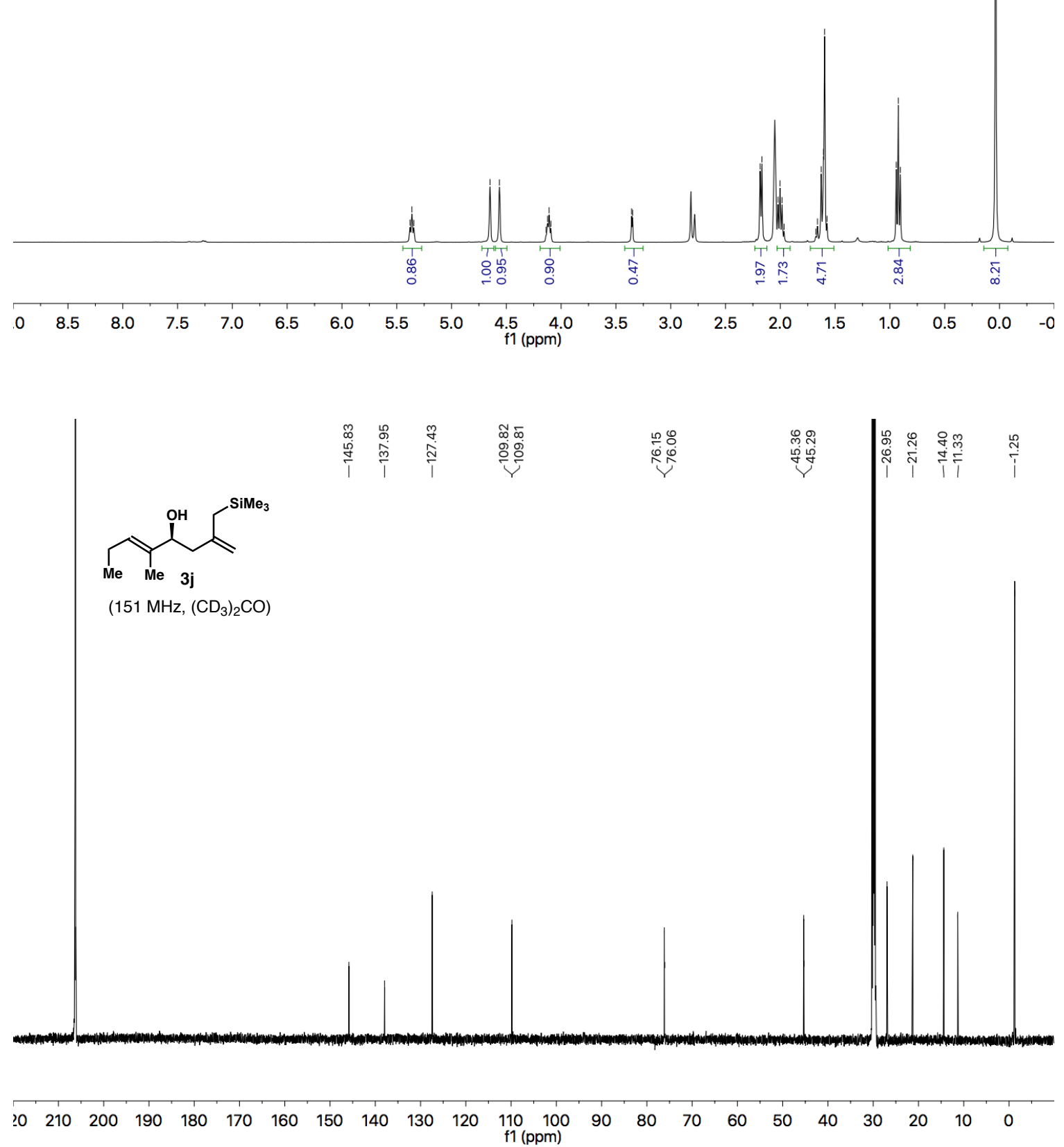

SI-21 

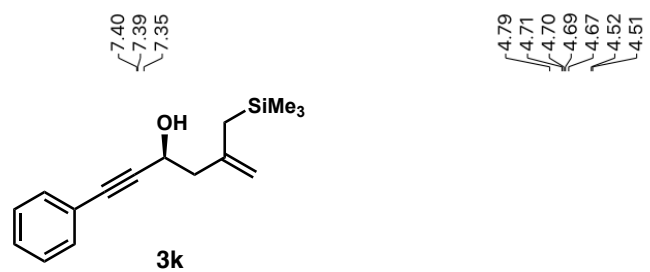

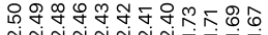

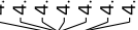

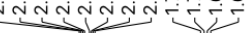

$\left(600 \mathrm{MHz},\left(\mathrm{CD}_{3}\right)_{2} \mathrm{CO}\right)$
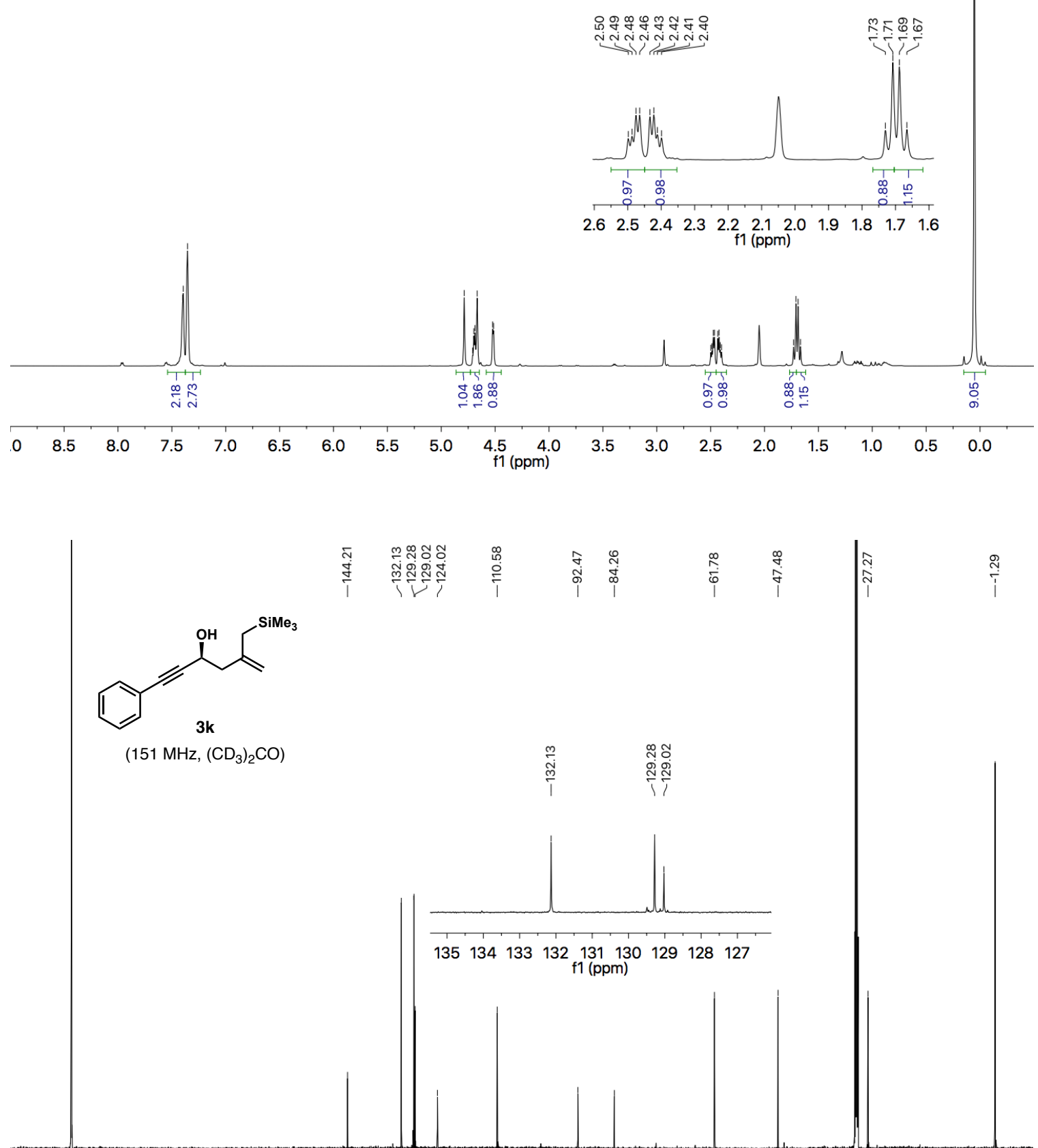

$\begin{array}{lllllllllllll}l 0 & 210 & 200 & 190 & 180 & 170 & 160 & 150 & 140 & 130 & 120 & 110 & 100\end{array}$

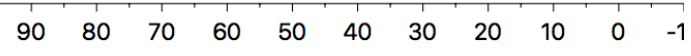



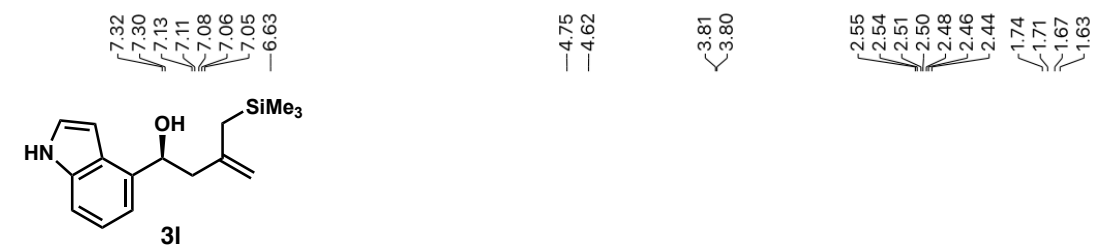

(400 MHz, $\left.\left(\mathrm{CD}_{3}\right)_{2} \mathrm{CO}\right)$
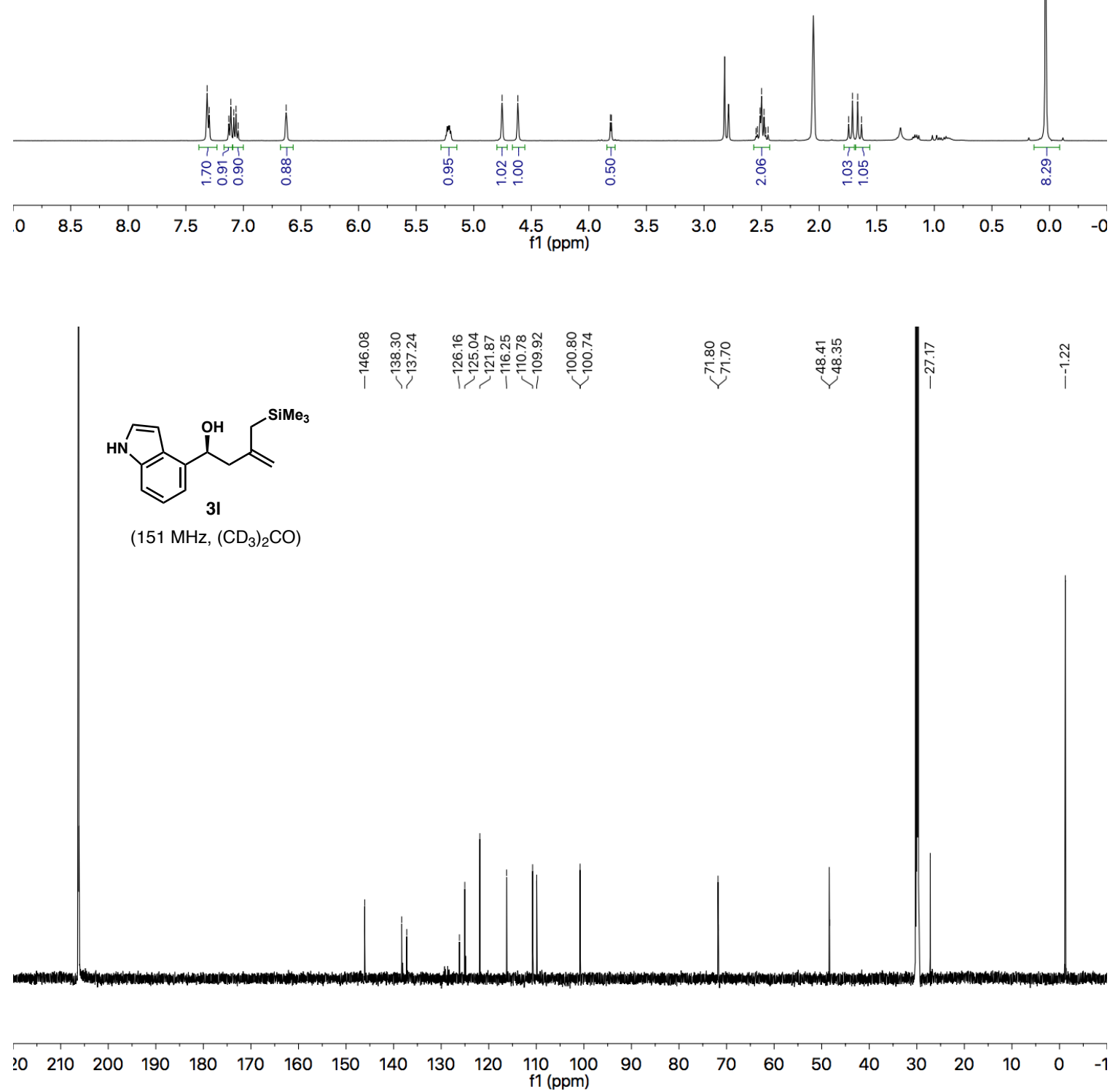

SI-23 

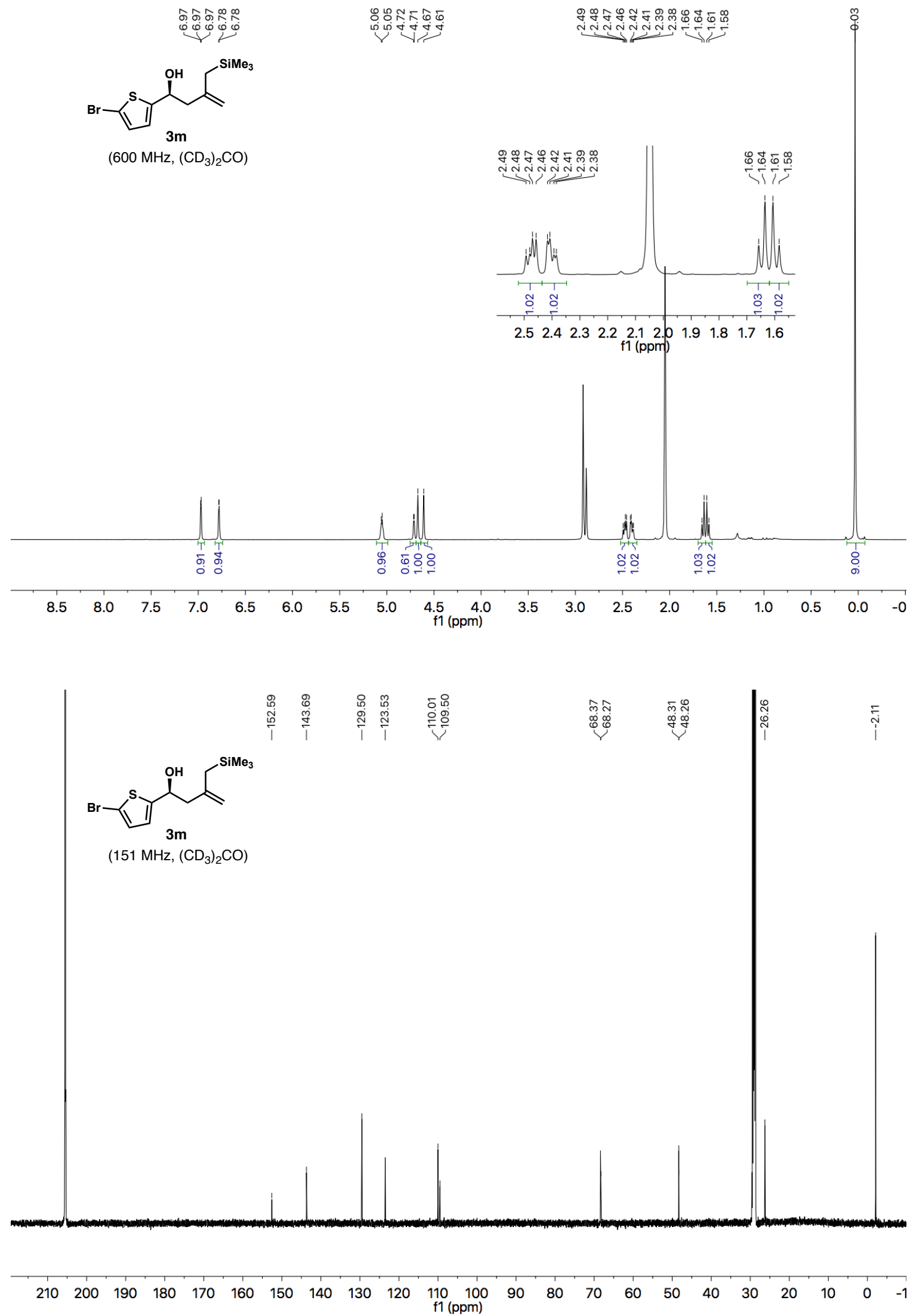

SI-24 

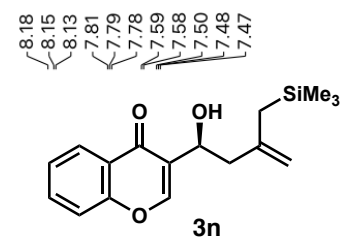

$\left(600 \mathrm{MHz},\left(\mathrm{CD}_{3}\right)_{2} \mathrm{CO}\right)$
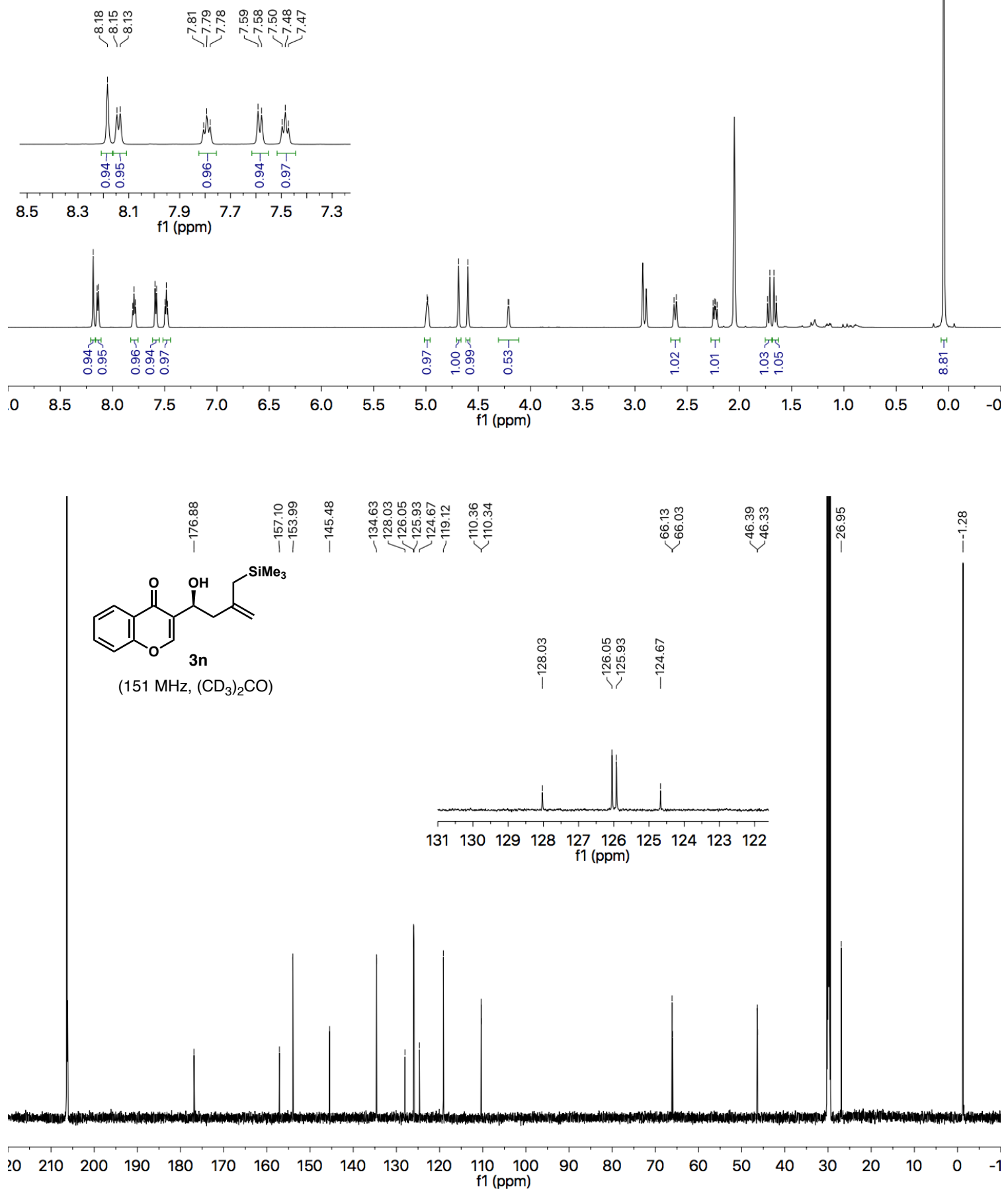

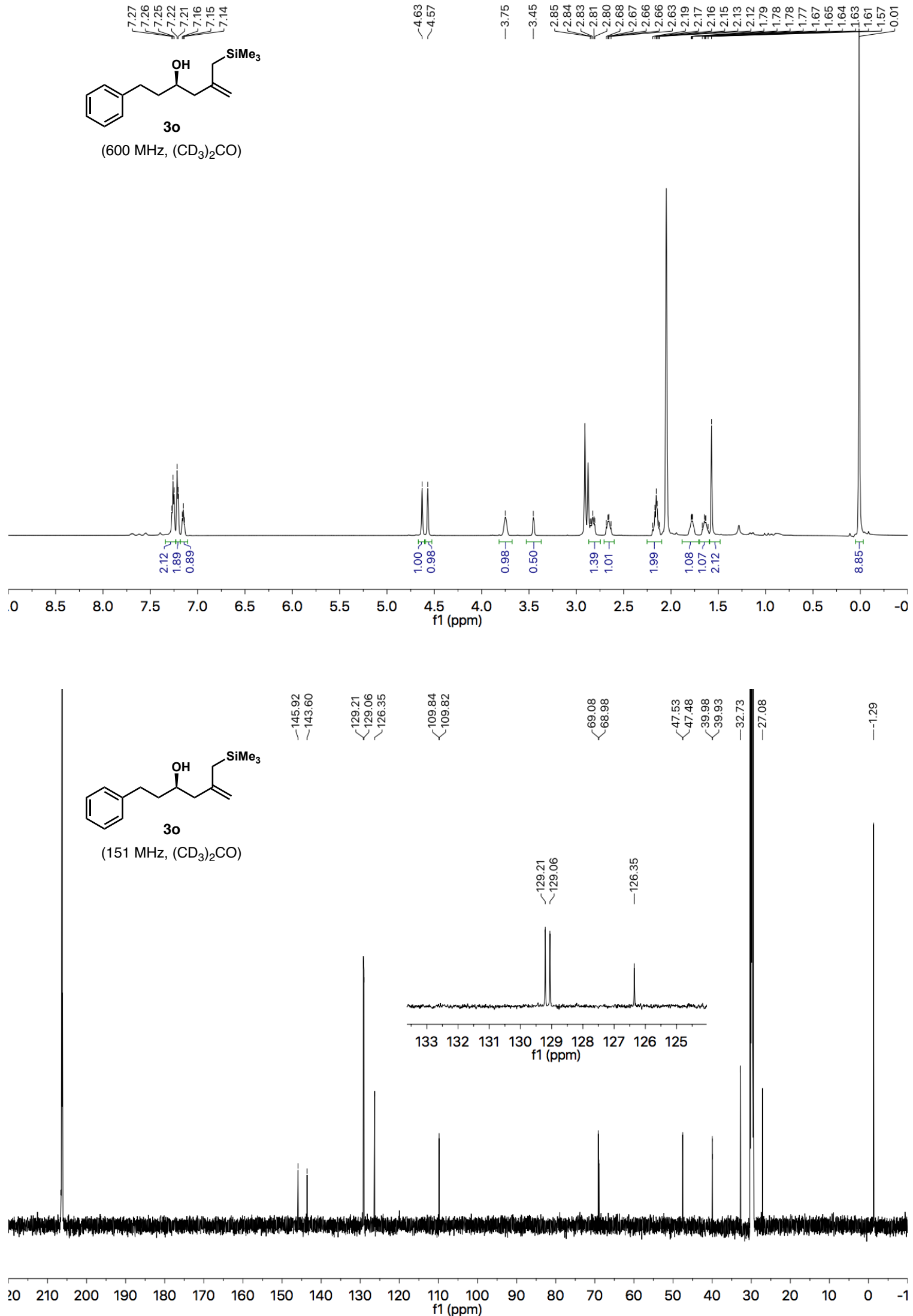


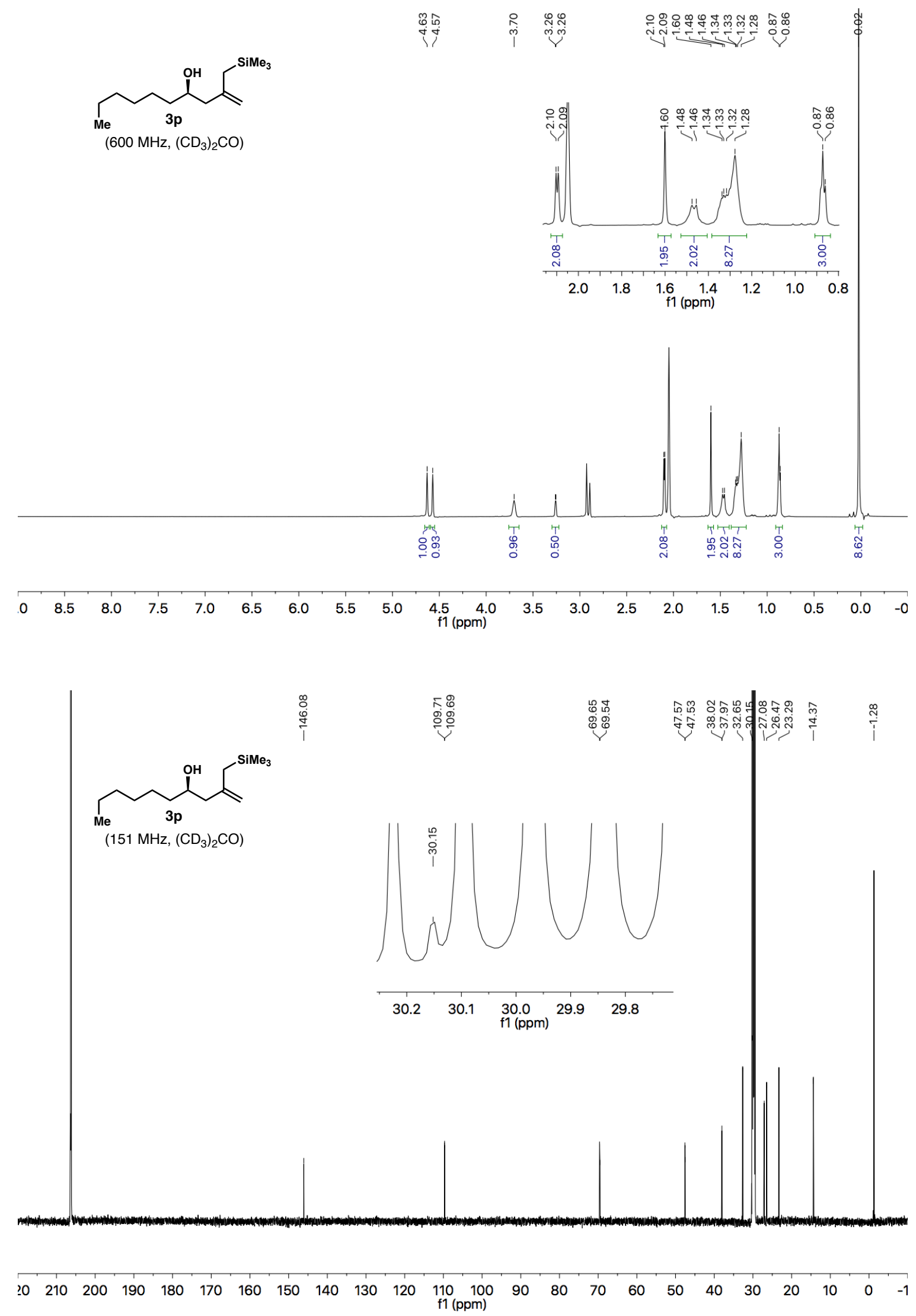

SI-27 


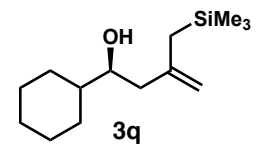

(600 MHz, $\left.\left(\mathrm{CD}_{3}\right)_{2} \mathrm{CO}\right)$
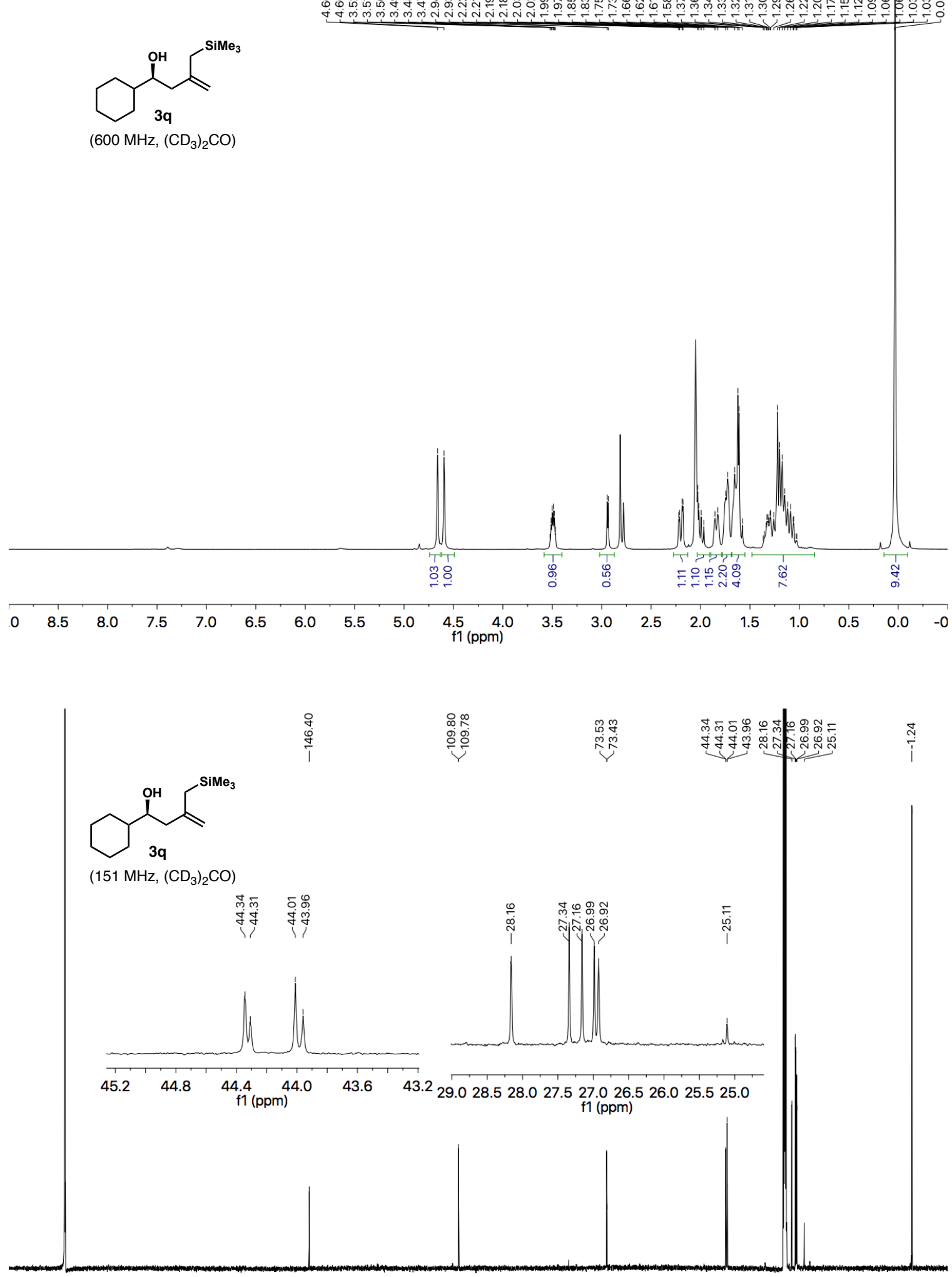

$\begin{array}{lllllllllllllllllllllll}20 & 210 & 200 & 190 & 180 & 170 & 160 & 150 & 140 & 130 & 120 & 110 & 100 & 90 & 80 & 70 & 60 & 50 & 40 & 30 & 20 & 10 & 0\end{array}$ 

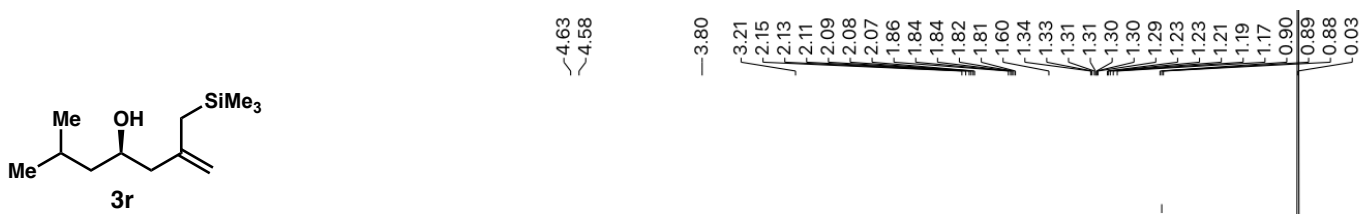

$\left(600 \mathrm{MHz},\left(\mathrm{CD}_{3}\right)_{2} \mathrm{CO}\right)$
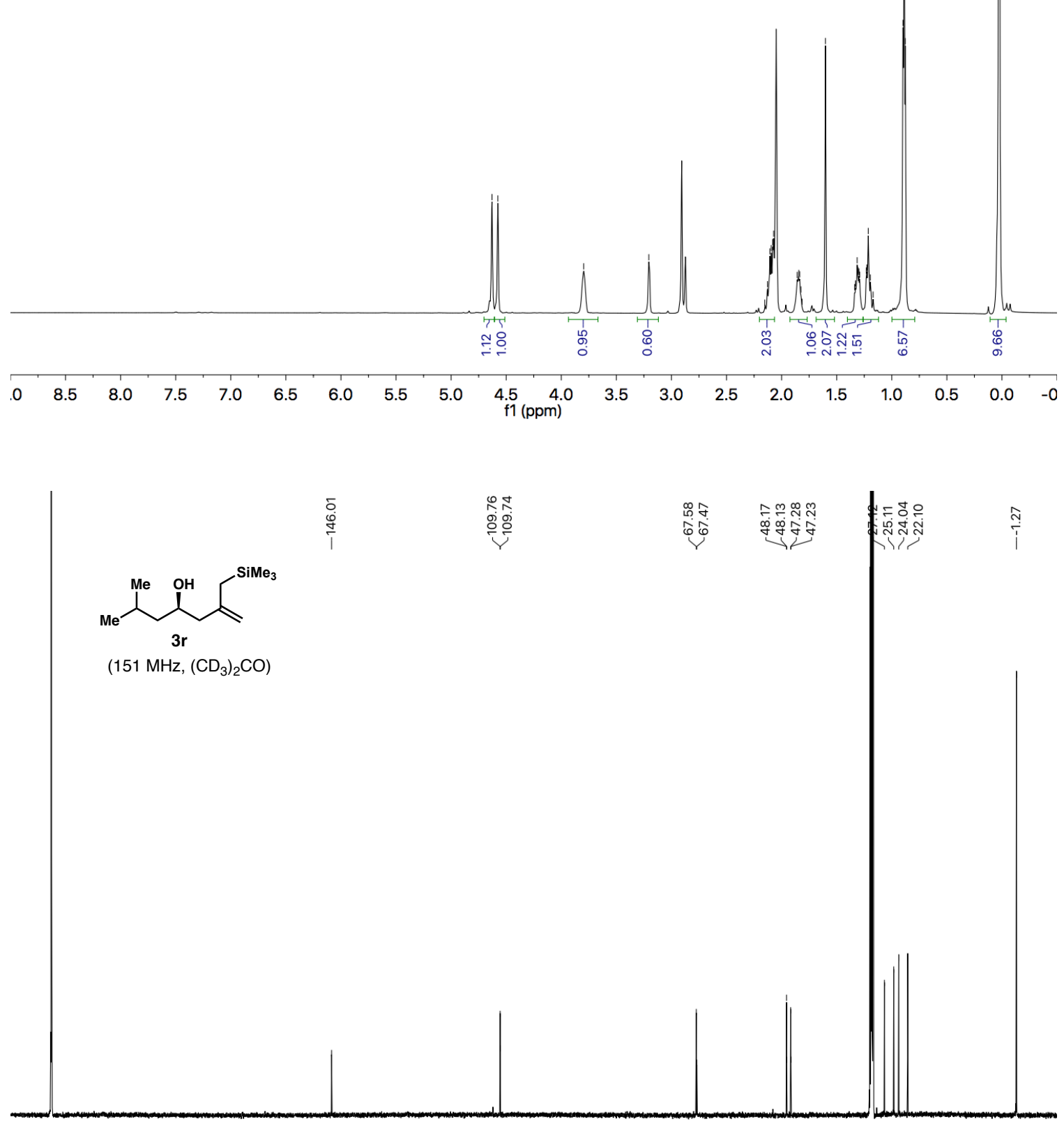

$\begin{array}{lllllllllllllllllllllll}210 & 200 & 190 & 180 & 170 & 160 & 150 & 140 & 130 & 120 & 110 & 100 & 90 & 80 & 70 & 60 & 50 & 40 & 30 & 20 & 10 & 0 & -1\end{array}$ 

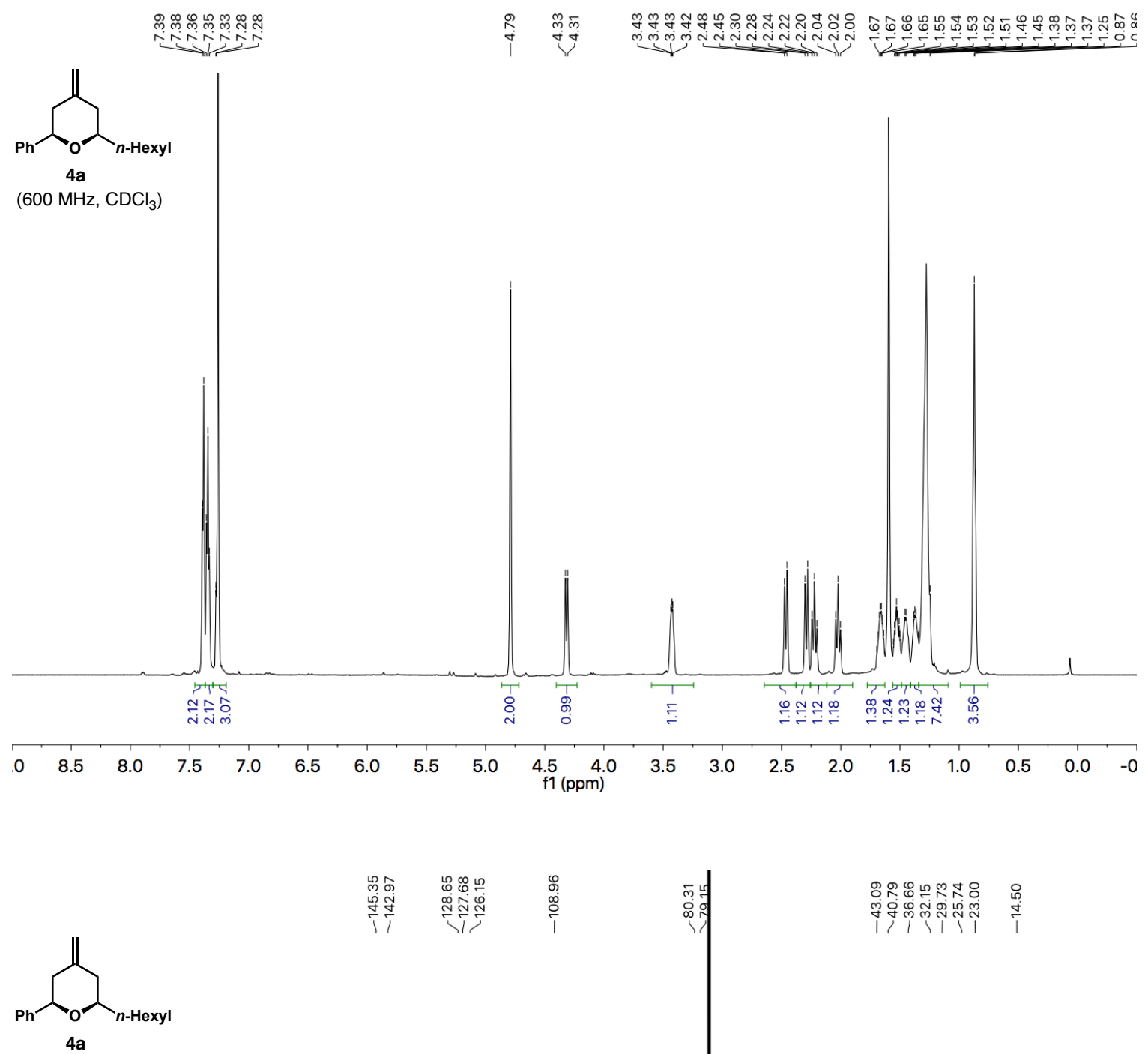

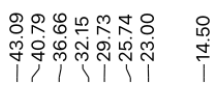

(151 MHz, $\mathrm{CDCl}_{3}$ )

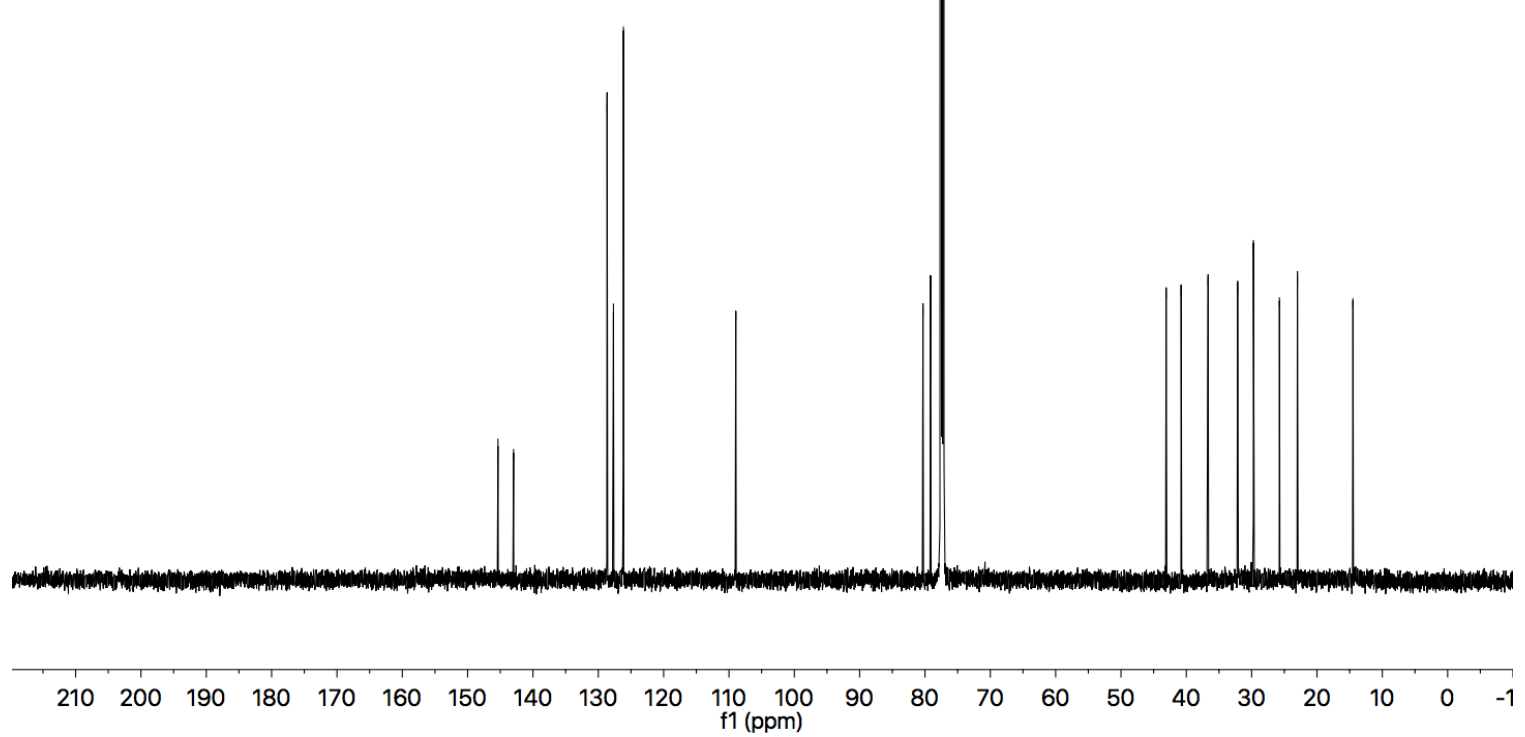

SI-30 


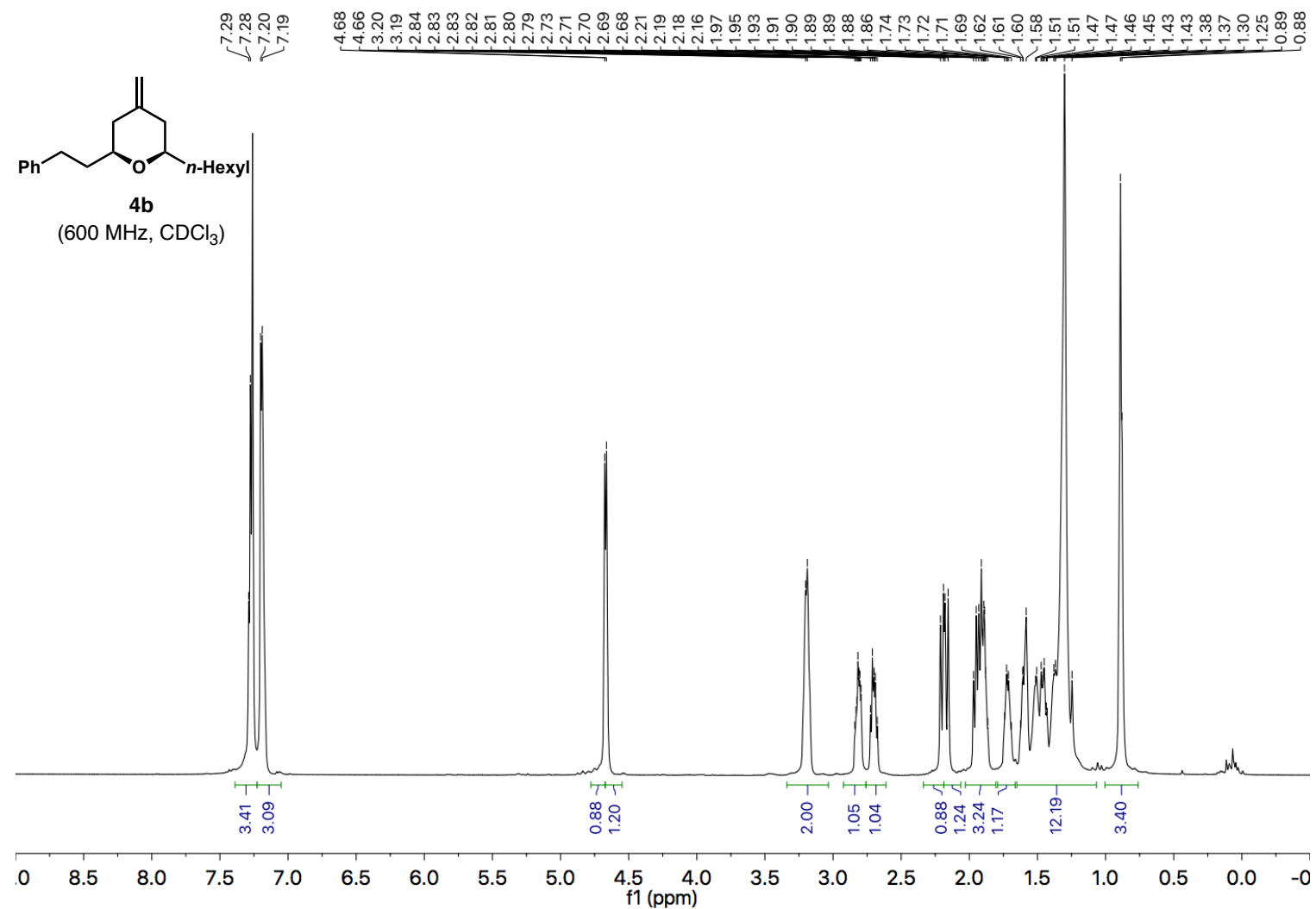

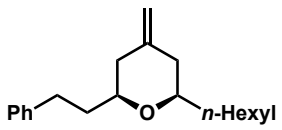

4b

(151 $\mathrm{MHz}, \mathrm{CDCl}_{3}$ )

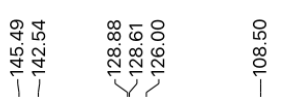

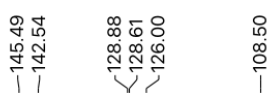

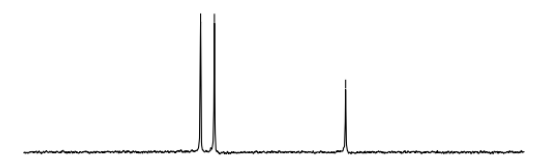

$\begin{array}{llllllllll}132 & 131 & 130 & 129 & 128 & 127 & 126 & 125 & 124 & 123\end{array}$ f1 (ppm)

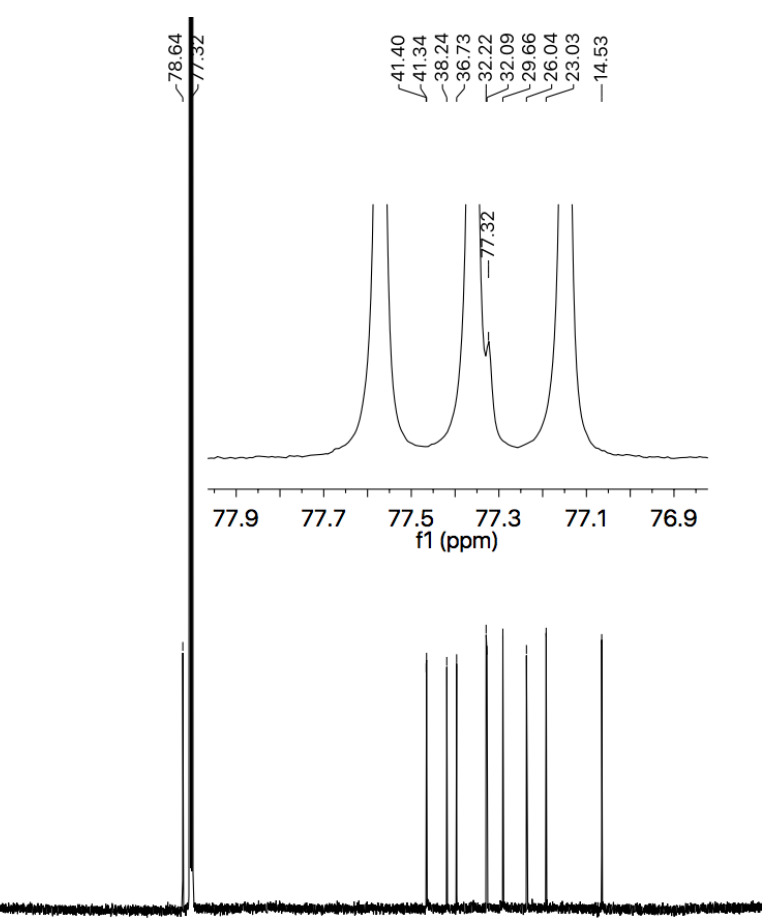

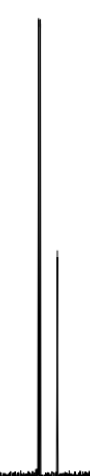

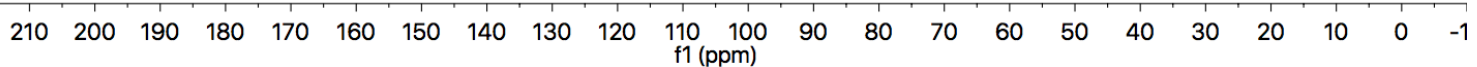



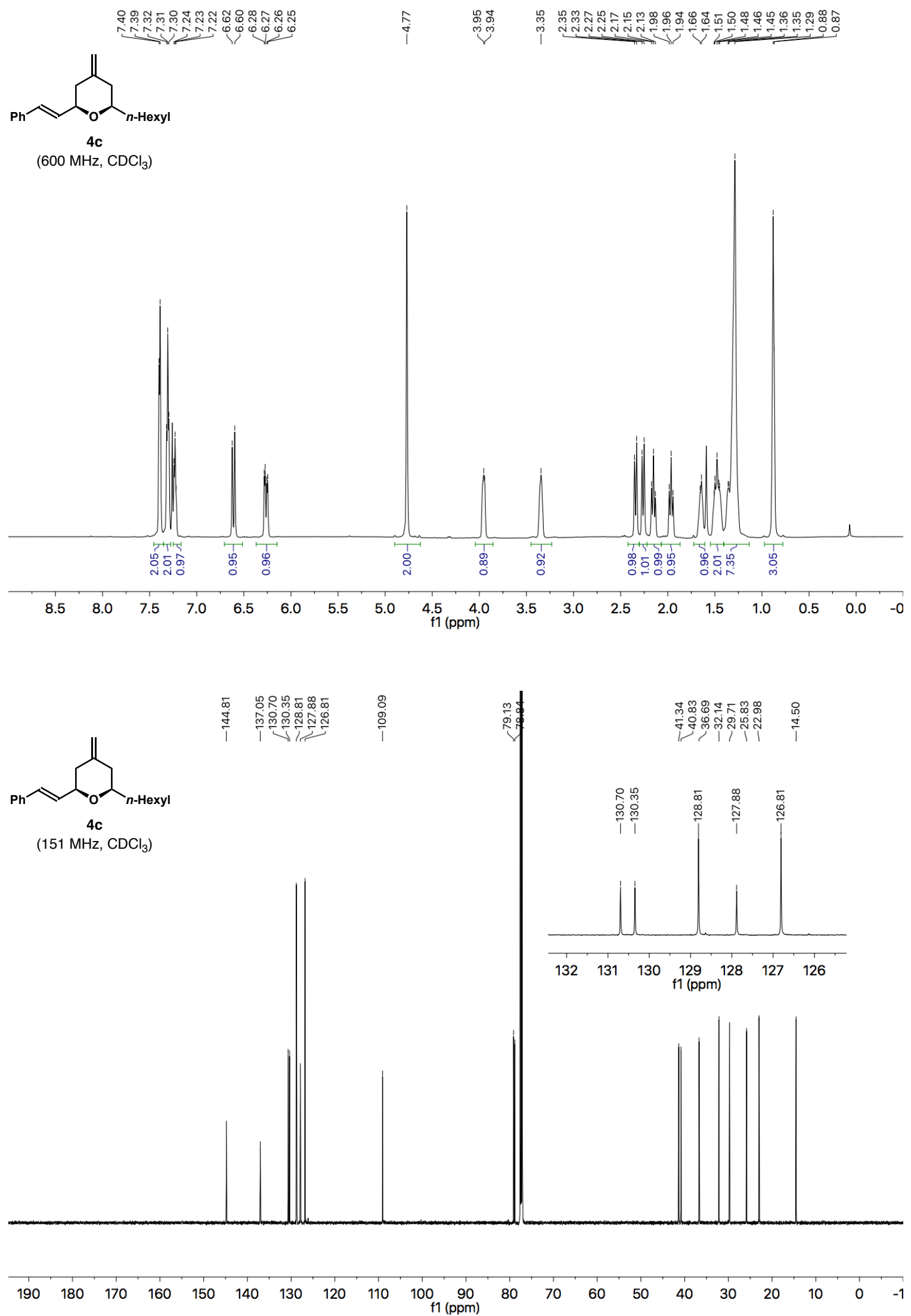

SI-32 

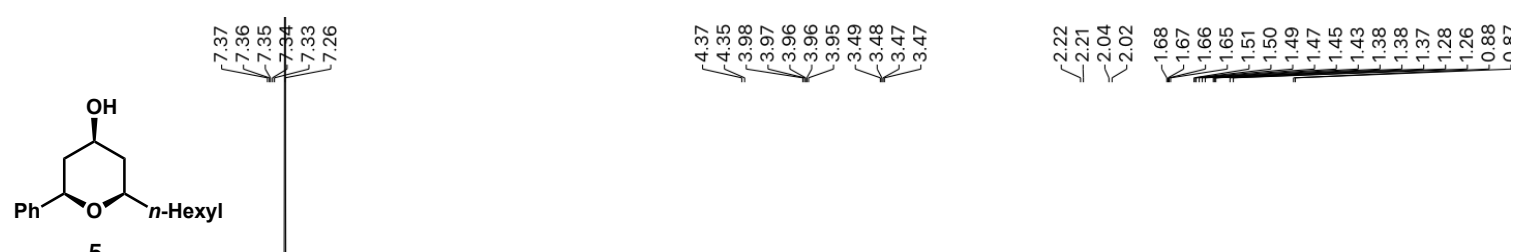

$\left(600 \mathrm{MHz}, \mathrm{CDCl}_{3}\right)$
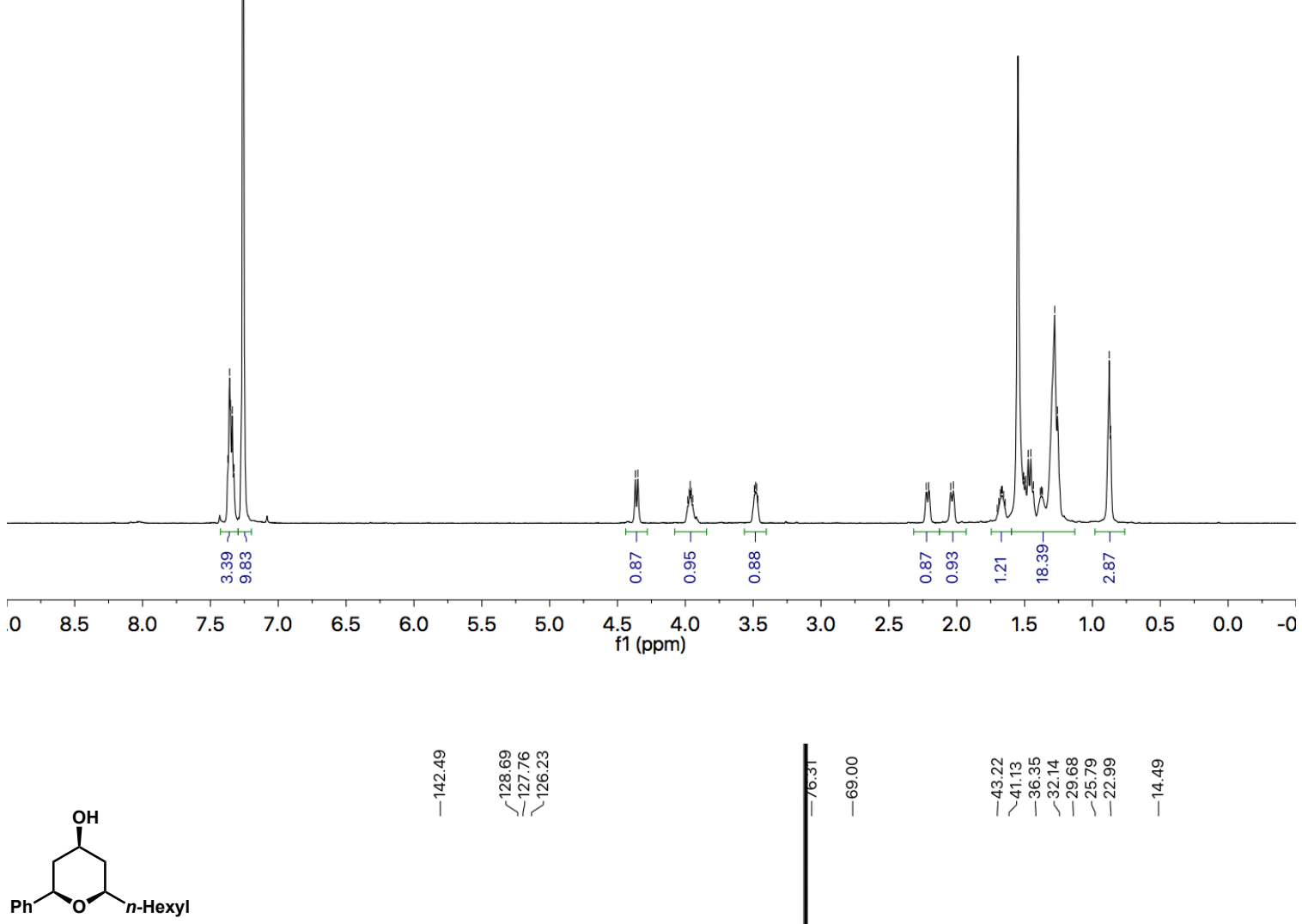

(151 MHz, $\mathrm{CDCl}_{3}$ )

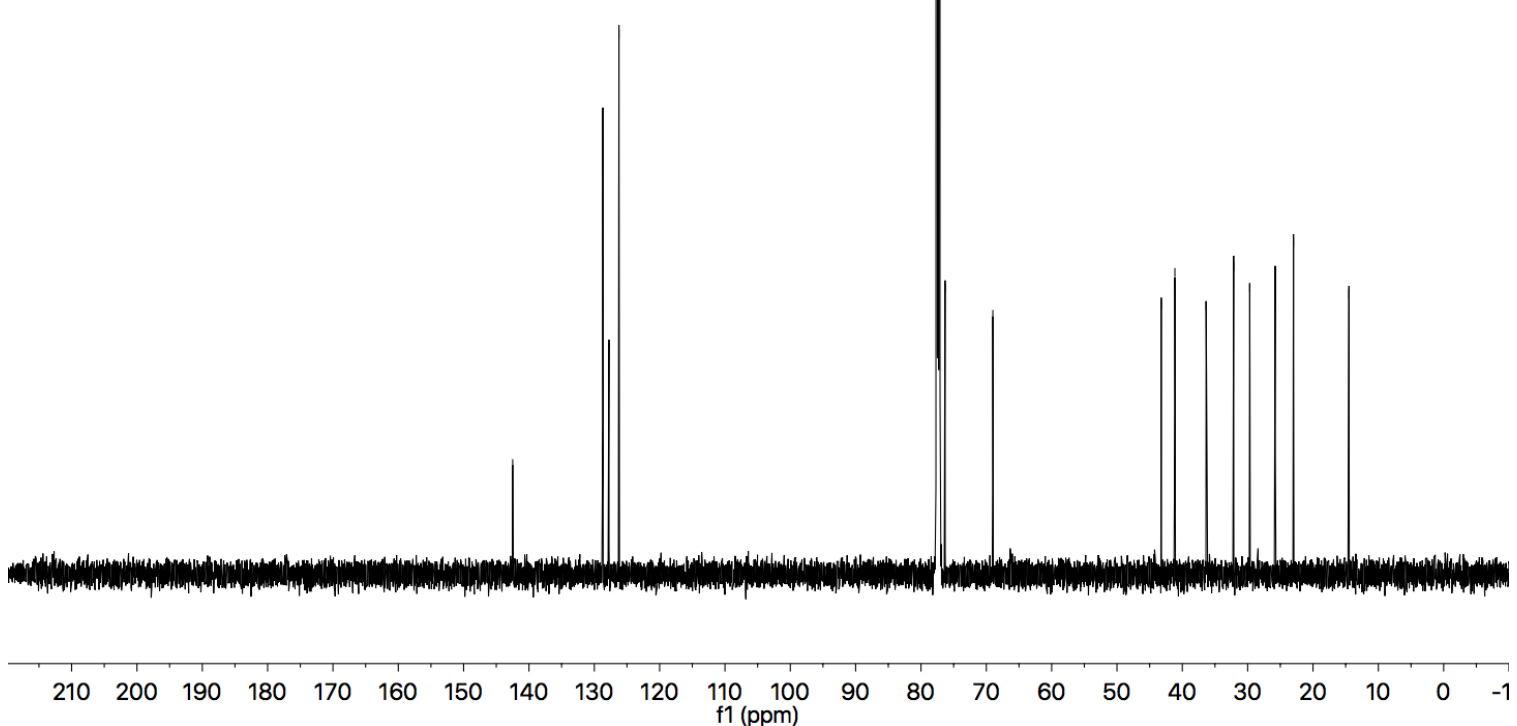




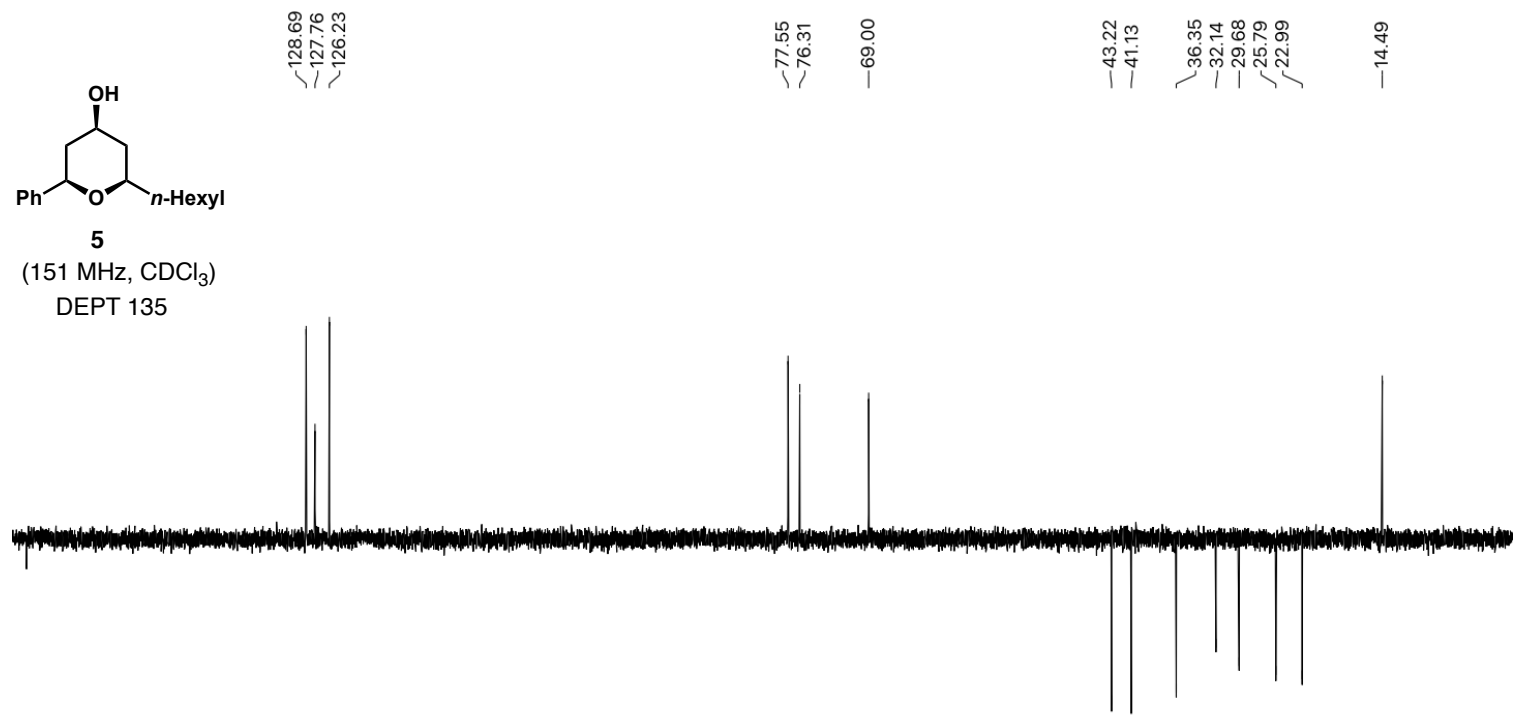

\begin{tabular}{|c|c|c|c|c|c|c|c|c|c|c|c|c|c|c|}
\hline 150 & 140 & 130 & 120 & 110 & 100 & 90 & $\begin{array}{c}80 \\
\mathrm{f} 1(\mathrm{ppm})\end{array}$ & 70 & 60 & 50 & 40 & 30 & 20 & 10 \\
\hline
\end{tabular}

SI-34 